\title{
Effets de la pratique de la pleine conscience et du Tai Chi Chuan sur la santé mentale d'étudiants : une étude pilote contrôlée non randomisée
}

\section{Effects of mindfulness and Tai Chi Chuan practice on mental health of students: A non-randomised controlled pilot study}

\author{
Philippe Lestage, Rong Xu
}

Laboratoire FRED, EA 6311, faculté des lettres et des sciences humaines, université de Limoges, 87000 Limoges, France

Mots clés : Pleine conscience ; Tai Chi Chuan ; Etudiants ; Santé mentale

Keywords : Mindfulness ; Tai Chi Chuan ; Students ; Mental health

Résumé De nombreuses recherches établissent les bienfaits de la pratique de la méditation de pleine conscience (mindfulness) et de celle du Tai Chi Chuan, mais très peu les mettent en perspective et portent sur des populations générales d'étudiants. Nous avons entrepris une étude pilote dans ce sens auprès de 33 étudiants de l'Université de Limoges inscrits dans trois stages de $18 \mathrm{~h}$ chacun, mindfulness, Tai Chi Chuan et témoin, organisés sur huit semaines. Les bénéfices ont été évalués trois fois (pré-/post-traitement, suivi à deux mois), aux mêmes moments pour les trois groupes, sur huit échelles explorant les difficultés de santé mentale et la santé mentale positive : questionnaire général de santé (GHQ-28), échelle de stress perçu (PSS-14), double échelle HAD anxiété-dépression, échelle d'auto-efficacité, échelle de satisfaction de vie (SWLS), échelle de bien-être (W-BQ12), questionnaire de mindfulness (FMI-14). En raison de quelques différences des niveaux de base, les échantillons des groupes mindfulness et Tai Chi sont considérés comme non équivalents. L'étude est quasi expérimentale avec groupe témoin en liste d'attente. Les résultats montrent des effets bénéfiques du Tai Chi Chuan, ceux de la pleine conscience n'apparaissent qu'à la mesure du temps de pratique personnelle quotidienne engagé, corrélés avec celui-ci. Une part des bénéfices se maintient deux mois après le stage.

\section{Summary}

Introduction. - This pilot study explores and provides insight into the effects of a Mindfulness Based Intervention (MBI) and Tai Chi Chuan practice on students of all disciplines at the University of Limoges (France). The students had the choice of enroling in one of three groups (mindfulness, Tai Chi Chuan or 'control') for an 18-hour course held over eight weeks at the Institut Universitaire de Formation des Maitres (IUFM). Tai Chi Chuan is a Chinese martial art from Tao and combines strength and flexibility, speed and slowness, active relaxation and concentration. It is often regarded as a form of 'moving meditation'. Its psychological and physiological benefits are widely recognised. Introduced by Jon KabatZinn at the University of Massachusetts through the Mindfulness-Based Stress Reduction (MBSR) programme, mindfulness has its roots in Buddhist meditation but is a secular program. MBIs were developed, as a continuation of MBSR, for clinical populations suffering from psychological and somatic health problems, and for non-clinical populations 
exposed to stress or simply looking for improved balance and well-being. Little research has been done into the benefits of Tai Chi Chuan or mindfulness for students, and it is rare to find research which compares the benefits of Tai Chi Chuan with those of meditation. However, students make up a vulnerable population exposed to stress and psychopathological disorders.

Method. - Thirty-three volunteer students chose between two course periods: JanuaryFebruary for the two mindfulness or Tai Chi Chuan experimental groups, March-April for the third waiting list control group. The benefits for the three groups were assessed pre- and posttreatment, and at two-month follow-up, by eight scales exploring mental health problems and positive mental health: General Health Questionnaire GHQ-28, Perceived Stress Scale PSS14, Hospital Anxiety and Depression (HAD) Scale, General Self-Efficacy Scale, Satisfaction With Life Scale (SWLS), Well-Being Scale (W-BQ12) and Freiburg Mindfulness Inventory (FMI-14). With regard to observed base level differences, samples of the Tai Chi and mindfulness groups were considered to be non-equivalent. The study was quasi-experimental controlled with pre-/post- and two-month follow-up testing, and a waiting list control group.

Results and Discussion. - University exams appear to have affected the pre-test health and stress scores in January to a certain extent. In fact, the comparisons with the Student test $t$ show a significant improvement in the control group between the pre-test at the beginning of January (during the exam period) and the post-test at the end of February (outside the exam period) on the GHG-28 $(P=0.044)$ and PSS-14 $(P=0.022)$ scales. The Tai Chi group recorded very significant pre- and post-test benefits on these two scales: GHG-28 $(P=0.008)$ and PSS-14 $(P=0.003)$. The Tai Chi group also noted significant benefits on the two other mental health problem scales : the HAD anxiety and HAD depression $(P=0.025 ; P=0.015)$. Very significant benefits were observed for this group on three scales of positive mental health: General Self-Efficacy Scale $(P=0.001)$, SWLS $(P=0.001)$, WBQ12 $(p=0.003)$. The control group recorded no significant pre-/post-test benefit on these five scales. Mindfulness practice did not produce any significant pre-/post-intervention benefits for the group as a whole, however, individual benefits were noted in correlation to the time of personal practice at home required during the eight weeks of the course. There was a weak correlation between daily practice time and improvement of general health GHQ $(r=0.558 ; P<0.1)$, medium correlation with anxiety reduction HAD $(r=0.619 ; P=0.056)$, strong correlation with stress reduction PSS $(r=0.759 ; P=0.011)$ and the development of aptitude in mindfulness FMI $(r=0.759 ; P=0.006)$. No correlation with the daily practice time was observed in the Tai Chi group. As a whole, the mindfulness group gained very significant benefits at the twomonth follow-up on the Anxiety (HAD anxiety, $P=0.005$ ) and Well-Being (WBQ12, $P=0.004)$ Scales, but not at post-test at the end of the course. The control group did not obtain benefits post-test. Ruths et al. (2013) similarly observed a decrease in anxiety (trait anxiety, STAI), three months after the course, rather than at the end of the course, in correlation with personal practice time up to the three months. They noted an improvement in well-being (psychological well-being, GHQ-12) from the end of the course, which lasted during the three month follow-up period, in correlation to the respect of daily personal practice. Fairly strong observance of required daily personal practice was noted in the Tai Chi (15 min) and mindfulness (23 min) groups. Tai Chi was clearly the most popular course, receiving a mark of 8.95/10. Mindfulness received more mixed reviews and was awarded an average mark of just 5.67/10. Tai Chi, as a more dynamic 'moving meditation', is possibly more suitable for young people, whereas mindfulness may be more beneficial for mature people. Incidentally, mindfulness students who committed to serious daily personal practice also gained significant benefits. 


\section{Introduction}

Les pratiques traditionnelles anciennes d'épanouissement intérieur telles que la méditation de pleine conscience (mindfulness) et le Tai Chi Chuan, exercées sous des formes modernes, paraissent favoriser la gestion du stress, équilibrer et améliorer la santé mentale. Leurs bienfaits s'observent notamment dans la population étudiante, fréquemment exposée à des conditions de vie difficiles et au stress. La mindfulness et le Tai Chi Chuan font l'objet de beaucoup de recherches mais généralement séparées, pourtant le Tai Chi Chuan est réputé être une forme de méditation en mouvement. Il apparaît donc heuristique d'explorer sur la base d'un même protocole la nature et l'importance des bénéfices recueillis de la pratique de ces deux disciplines respectives par des étudiants.

Nous avons engagé dans ce sens une étude pilote examinant la faisabilité et le bénéfice de l'investissement d'étudiants de tous horizons disciplinaires dans des programmes de pleine conscience (intervention basée sur la pleine conscience [IBPC]) et de Tai Chi Chuan (style Chen) proposés en $18 \mathrm{~h}$ sur huit semaines à 33 étudiants de l'université de Limoges. L'étude explore une variété de mesures concernant la réduction des difficultés mentales et le développement des états mentaux positifs. Elle est quasi expérimentale, contrôlée mais non randomisée, avec pré-/post-test et suivi à deux mois, groupe témoin en liste d'attente. Du fait de certaines différences observées dans les niveaux de base de leurs évaluations prétraitement et en termes d'âge, les groupes mindfulness et Tai Chi ne peuvent être comparés et sont considérés comme non équivalents.

\section{Stress et souffrance psychique des étudiants}

Les étudiants constituent une population fragile, vulnérable, potentiellement exposée à la précarité, au stress et davantage sujette aux troubles psychiques que les jeunes non étudiants et les adultes. Une étude épidémiologique conduite en 2005-2006 sur des étudiants de première année de six universités du sud-est de la France a montré la prévalence des troubles suivants : troubles anxieux $(15,7 \%)$, dépression caractérisée $(8,9 \%)$, troubles liés à l'usage de substances $(8,1 \%)$ [1]. La « troisième enquête nationale sur la santé des étudiants » menée en France en 2011 pointe du doigt le mal-être et la précarité : sentiment constant de tristesse et de déprime (38\%), idées suicidaires (12\%), moins de 400 euros de revenus par mois (près de $50 \%$ ), nécessité d'exercer une activité rémunérée en parallèle $(28 \%) \ldots$ Le stress est lié aux rythmes de travail, aux emplois du temps décousus, aux transports, aux exigences de performance, aux examens [2]. Les étudiants des champs de la santé (médecine, soins infirmiers, chirurgie dentaire...), devant fournir un important "travail émotionnel», apparaissent spécialement stressés, exposés à la détresse psychologique, au burnout et font l'objet de très nombreuses recherches (plus de 600 articles répertoriés par Shapiro et al. en $2000[3])$.

\section{Les bienfaits de la pratique de la pleine conscience et du Tai Chi Chuan}

La mindfulness ou pleine conscience a été introduite il y a une trentaine d'années par Jon Kabat-Zinn à l'Université du Massachusetts. Son programme de mindfulness-based stress reduction (MBSR) ou réduction du stress basée sur la pleine conscience était destiné à des patients affectés de maladies chroniques insuffisamment traitées par la médecine, susceptibles de trouver en elle un complément pour soulager leurs douleurs et leur stress [4,5]. La MBSR s'est ensuite répandue en Amérique puis en Europe, et sa pratique bénéfique a été élargie à de nombreux domaines psychologiques et psychopathologiques. 
La mindfulness est une adaptation occidentalisée de la méditation bouddhique. Elle développe une attitude d'attention dénuée de jugement à l'expérience vécue dans l'instant présent et aux processus physiques et mentaux alors engagés (pensées, émotions, sensations corporelles) ; dans les divers contextes de la vie courante. Le déploiement de cette prise de conscience dans le moment présent et l'acceptation de l'expérience rencontrée peuvent réduire les effets de stress associés à une orientation excessive vers le passé (les ruminations en particulier) ou le futur (les soucis en particulier) notamment lorsque le stress est lié à la dépression ou l'anxiété. Cet engagement expérientiel conscientisé se démarque des stratégies réflexes habituelles d'évitement qui ne font que renforcer les états intérieurs redoutés. Il favorise une meilleure perception de la réalité rencontrée à la place de la réactivité émotionnelle et mentale immédiate qui s'élève habituellement à travers les filtres inconscients du psychisme. La pleine conscience est réputée atténuer le stress, les affects négatifs et favoriser l'épanouissement de qualités positives telles que le bien-être, la vitalité, les capacités d'adaptation [6-11].

Les recherches investiguant de manière approfondie les «mécanismes» potentiels d'action de la pleine conscience en sont à leur début, mais croissent en nombre exponentiel [12]. Tout en soulignant la complexité des processus engagés, elles privilégient les suivants : attention dirigée vers l'expérience présente vécue, dans une attitude d'acceptation ouverte, sans jugement ni résignation, une attitude bienveillante, d'auto-compassion; régulation émotionnelle par exposition et réévaluation positive, réduction de la réactivité cognitive et des ruminations; décentration métacognitive (meta-awareness) éveillant un changement de perspective sur le soi (the self), flexibilité cognitive, émotionnelle et comportementale, gestion de soi; relaxation [11,13-18]. De récentes avancées mettent en évidence le rôle essentiel des mécanismes de contrôle attentionnel ainsi que les caractéristiques spécifiques des activités neuronales qui les sous-tendent et des zones cérébrales suractivées (techniques d'EEG, d'imagerie cérébrale, test de Stroop...) [19,20]. De telles recherches explorant la nature des mécanismes neuronaux impliqués sont en progression, certaines d'entre elles différencient leur étude en fonction de différents types d'activité méditative [16,21].

Au-delà de leur identification, la question se pose de savoir si ces divers mécanismes interviennent de manière équivalente selon la variété des populations, cliniques ou non cliniques, ce qui pour le moment semble être le cas [17]. Ajoutons enfin qu'à la différence de bien des approches thérapeutiques, les thérapies de pleine conscience insistent davantage sur la mobilisation des ressources personnelles que sur le traitement des problèmes spécifiques qui affectent la personne [22].

La MBSR a donné naissance à quelques pratiques voisines dans différents champs cliniques, au premier rang desquelles la mindfulness-based cognitive therapy (MBCT) ou thérapie cognitive basée sur la pleine conscience. Combinant la MBSR et la thérapie cognitive, la MBCT fut initialement conçue pour la prévention de la rechute dépressive [23] puis élargie à d'autres pathologies : épisodes dépressifs majeurs, trouble bipolaire, phobie sociale, trouble d'anxiété généralisée... Elle fait l'objet de nombreuses recherches universitaires visant à préciser les conditions de son efficacité $[9,24,25]$. La MBSR et ses nombreuses variantes utilisant à des degrés divers la pleine conscience sont fréquemment regroupées sous la dénomination de mindfulness-based interventions (MBI) ou interventions basées sur la pleine conscience (IBPC) et rattachées à la troisième vague " émotionnelle » des thérapies comportementales et cognitives [13,26]. 
Dans l'ensemble, les recherches rendent compte de bienfaits significatifs des MBIs sur le plan de la santé mentale mais pas clairement sur celui de la santé physique. Aussi bien pour les populations non cliniques exposées au stress ou en simple recherche de meilleur équilibre, de bien-être (well-being) et de qualité de vie [22,27-29], que pour les populations cliniques affectées de difficultés psychologiques et/ou somatiques: troubles anxieux, dépressifs, phobiques, obsessionnels, troubles du comportement alimentaire, réduction du stress et des troubles de l'humeur chez des patients souffrant de douleurs chroniques, de fibromyalgie, d'arthrite rhumatoïde, de cancer... [7,8,14,22,30-32]. La méditation de pleine conscience a aussi été introduite dans le champ éducatif. Pour réduire le stress des enseignants, les prémunir du burnout [33-35] et à destination des élèves, des enfants, des adolescents [3638]. Elle montre des effets positifs dans la gestion du stress des étudiants [39,40]. La grande majorité des recherches porte sur les étudiants des métiers de la santé (médecin, infirmier, dentiste...) qui doivent fournir un important «travail émotionnel », s'exposant à une réelle détresse psychologique et eux aussi au burnout [18,41-46]. De notre côté nous avons fait porter notre étude sur une population générale d'étudiants, issus d'horizons disciplinaires variés.

Les IBPC sont généralement pratiquées en groupes de dix à vingt personnes, pendant une vingtaine d'heures programmées sur huit semaines, à raison d'une séance par semaine. Cellesci sont composées d'exercices progressifs dérivés de la méditation, pour certains du yoga: balayage corporel (body scan), méditation assise posée sur différents supports (respiration, sensations corporelles, bruits, pensées, émotions...) ou sur une attention sans objet, marche en pleine conscience, régulation émotionnelle... Les participants sont invités à répéter quotidiennement chez eux ces exercices de «pratique formelle» et à les prolonger de " pratique informelle » consistant à rester présent à soi-même, dans la pleine conscience des activités ordinaires habituelles (déjeuner, se brosser les dents, se doucher...).

La popularité de la mindfulness s'est très largement accrue ces dernières années, on ne compte plus le nombre de sites internet proposant sa pratique. Sur le terrain scientifique la multiplication des recherches est également considérable, mais celles-ci progressent prudemment. Les bénéfices observés commencent à être mieux identifiés, analysés, délimités. Des études quantitatives évaluant l'impact du temps de pratique quotidienne personnelle sur ces bénéfices sont à présent engagées [18,45-47]. Une limite importante tient à la nécessité que les praticiens MBSR/MBCT disposent d'une solide formation et d'une pratique de plusieurs années de méditation [22]. Au moins trois ans de pratique personnelle, stages et retraites silencieuses pour le Center for Mindfulness de l'Université du Massachusetts - une formation semblable est proposée en France par l'Association pour le Développement de la Mindfulness $(\mathrm{ADM})-2000 \mathrm{~h}$ de méditation, soit presque deux ans à méditer $3 \mathrm{~h}$ par jour, sont recommandées aux formateurs par Chade-Meng Tan et Fabrice Midal [48-50]. Une augmentation des symptômes ou quelques effets secondaires indésirables (dépersonnalisation, détérioration de l'humeur...) peuvent parfois être observés [22,51,52]. Sur le plan épistémologique, l'exploration des correspondances et des synergies entre les deux univers historico-socio-culturels très différents dont relèvent le bouddhisme et les MBIs commence à être entreprise [53-55].

Le Tai Chi Chuan (ou Tai Ji Quan) est un art martial chinois fondé sur l'enchaînement de mouvements fluides et souples, pratiqués avec lenteur, harmonieusement, selon des séquences plus ou moins longues ou complexes. Il engage aussi parfois des mouvements rapides et explosifs. Ses racines historiques peuvent être associées à deux emblèmes de la civilisation chinoise, le taoïsme et le Livre des mutations (Yi Jing). Le Tai Chi Chuan 
recherche dans l'être humain lui-même, physiquement et mentalement, l'équilibre harmonieux entre les deux entités fondamentales, opposées et complémentaires, du yin et du yang.

Depuis son apparition, le Tai Chi Chuan a évolué en différents styles parmi lesquels le style Chen est le plus ancien, le style Yang est le plus populaire. Le style Chen est plus dynamique, il alterne des rythmes lents et actifs, des techniques « dures » et « douces », des mouvements spiralés, des sorties de force. Le style Yang est généralement composé de pratiques plus lentes et douces, de cercles gracieux.

Les enchaînements sont désignés par des appellations imagées telles que « la grue blanche déploie ses ailes ». La mise en oeuvre de la force est souple et dynamique et non pas brute ; elle requiert un relâchement des tensions musculaires et articulatoires dans la fluidité des mouvements et de leur coordination, en relation avec la respiration. La dynamique énergétique développée est semblable au mouvement ondulatoire des vagues, elle opère une circulation de l'énergie vitale, le « Qi ». L'équilibre s'enracine dans le sol (la Terre), à partir d'un positionnement attentif des pieds, des jambes, des appuis. Le pratiquant fait circuler l'énergie, la mobilise à son gré pour se détendre, se recentrer, se défendre. La tranquillité intérieure est acquise dans le mouvement, en une « relaxation active», fine et attentive. Le Tai Chi est souvent considéré comme une forme de « méditation en mouvement ».

L'étude du Tai Chi Chuan et de ses bienfaits peut être engagée selon deux grandes perspectives: traditionnelle et scientifique [56]. Sous l'angle traditionnel, la dynamique énergétique du Qi relève du paradigme classique de la médecine chinoise. Il ne nous appartient pas d'en discuter la pertinence, il importe en revanche de garder à l'esprit ce fondement culturel. Sous l'angle scientifique, il est possible d'examiner les effets physiques et psychologiques de la pratique du Tai Chi Chuan selon les méthodes de la science moderne. Notre recherche s'inscrit dans ce dernier cadre.

De nombreuses recherches ont été conduites par la communauté scientifique chinoise et occidentale pour évaluer les bienfaits du Tai Chi Chuan dans un très grand nombre de domaines liés à la santé physique, psychologique et à l'éducation. Leur nombre s'est accru de manière considérable depuis une trentaine d'années. À la différence des recherches entreprises sur la mindfulness (MBSR, MBCT, MBI), les programmes de pratique du Tai Chi ne sont pas standardisés. Il en résulte une grande variabilité des résultats selon les styles (Yang, Chen...), les objectifs poursuivis, la durée et la fréquence des pratiques... Malgré tout, des bénéfices significatifs sont observés dans la plupart des études [56-59].

Une grande part d'entre elles concerne des enjeux de santé physique tels que les fonctions cardiovasculaire et pulmonaire, la pression sanguine et l'hypertension, la fonction immunitaire, les douleurs chroniques, le sommeil, le sens de l'équilibre et la prévention des chutes, la souplesse articulaire et musculaire, la maladie de parkinson, la fibromyalgie [6062]. Sur le plan de la santé psychique, les études portent principalement sur les aspects de qualité de vie, bien-être, auto-efficacité, dépression, stress et troubles de l'humeur [63-71]. Il faut noter cependant un certain embrouillement dans la classification de ces variables psychologiques, les différents chercheurs les classant selon un large éventail de critères. Ainsi trouve-t-on assez indistinctement utilisés les termes de qualité de vie, de bien-être et de perception de la santé mentale, ou encore de dépression, d'anxiété et de stress [67].

Pour une large part, les recherches sont consacrées aux personnes âgées, le plus souvent à partir du style Yang et de formes simplifiées de celui-ci [72-74]. Peu d'études sont publiées 
sur les populations d'élèves de l'enseignement secondaire dans les revues scientifiques occidentales [75,76] et très peu sur les étudiants de l'enseignement supérieur [77]. Dans la littérature scientifique chinoise, en revanche, le nombre de publications les concernant est beaucoup plus important, mais le classement des revues chinoises ne suit pas les mêmes critères qu'en occident. Une quarantaine d'articles de premier rang («Core Journals in China ») ont pour objet la pratique du Tai Chi par les étudiants, les bénéfices qu'ils en retirent, dans le moteur de recherche chinois http://www.cnki.net [78-81].

Les études comparant le Tai Chi Chuan avec d'autres pratiques comme la relaxation, la méditation, sont peu nombreuses [82-84]. Celles qui portent spécifiquement à la fois sur le Tai Chi et la mindfulness (de type MBSR, MBCT, MBI) sont très rares et se limitent généralement à l'association complémentaire des deux pratiques dans les mêmes programmes. Nous n'avons pas trouvé de recherches contrôlées comparant quantitativement les bénéfices respectifs des pratiques du Tai Chi et de la mindfulness dans la littérature scientifique occidentale et chinoise. Plus largement, nous n'avons trouvé aucune publication chinoise ayant à la fois pour objet la pratique du Tai Chi et celle de la méditation en général, ou de la mindfulness («zheng nian») en particulier, sur les moteurs de recherche chinois tels que http://www.baidu.com, http://www.cnki.net ou les sites documentaires tels que http://www.wanfangdata.com.

La mindfulness fait l'objet d'importantes recherches en Chine. Principalement consacrées à la MBSR et la MBCT, mais aussi plus largement à d'autres approches de la «troisième vague " des TCC telles que l'ACT (Acceptance and Commitment Therapy) et la DBT (Dialectical Behavior Therapy). En chinois le mot mindfulness correspond à "zheng nian », qui ne dérive pas du terme anglais mindfulness mais des racines originelles sanskrites « samyak » (juste, pour « zheng ») et « smrti » (attention, pour « nian »). Zheng nian (chin.) est donc « samyak smrti » (sk.), l' « attention juste», traduit en anglais par mindfulness et en français par pleine conscience. Les seules occurrences des mots "zheng nian » (au sens occidental de la mindfulness MBSR, MBCT, MBI) dans les moteurs de recherche chinois sont très nombreuses : nous recensons près de 70 articles de premier rang écrits en chinois comprenant ces deux mots clefs pour la seule période 2012-2015 sur http://www.cnki.net [85-90].

\section{Objectifs de l'étude}

Le Tai Chi Chuan étant réputé être une forme de "méditation en mouvement ", il nous est apparu judicieux de développer une recherche évaluant les bienfaits respectifs du Tai Chi Chuan et de la méditation de pleine conscience (IBPC). A notre connaissance une mise en perspective des bénéfices de ces deux pratiques n'a pas encore été entreprise, tant en occident qu'en Chine, sur quelque population que ce soit : clinique, non-clinique, enfants, étudiants, adultes, personnes âgées... Concernant les étudiants, très peu de recherches ont été effectuées dans le monde occidental pour explorer les bienfaits de la pleine conscience, très peu également pour ceux du Tai Chi ; leur nombre est plus important en Chine. Notre étude pilote s'engage dans le sens de cette mise en perspective des bénéfices des deux pratiques. Nous avons cherché à explorer et évaluer les bienfaits que peuvent procurer la pratique de la méditation de pleine conscience et celle du Tai Chi Chuan à une population générale d'étudiants de tous horizons disciplinaires. Nous faisons l'hypothèse de l'obtention de ces bienfaits à la fois sur le versant de la réduction des difficultés mentales, notamment du stress, et sur le versant opposé de la santé mentale positive, généralement associée au bien-être 
subjectif, à la qualité de vie dans le champ de la psychologie positive. Nous explorons un assez large éventail de ces bénéfices potentiels, sur la base de sept échelles dont une dédoublée. Nous faisons également l'hypothèse d'une corrélation entre les bénéfices acquis et le temps de pratique de pleine conscience ou de Tai Chi effectué quotidiennement à domicile pendant la durée des stages.

Souhaitant nous rapprocher de la réalité clinique du terrain, nous avons ouvert un stage de mindfulness et un stage de Tai Chi Chuan à l'ensemble des étudiants de l'Université de Limoges et les avons laissés s'inscrire librement, à la période de leur choix, dans l'un des trois groupes Tai Chi Chuan ou mindfulness (pendant la même période) ou témoin (en liste d'attente). Dans ces conditions de recherche clinique ajustée au terrain [91], non randomisée, notre protocole est quasi expérimental. La comparaison des groupes mindfulness et Tai Chi, envisagée au départ, ne s'est pas avérée possible au regard des différences de niveaux de base observées sur certaines des échelles de mesures et des différences d'âge moyen des trois groupes. Les échantillons pleine conscience et Tai Chi sont donc considérés comme non équivalents. Les huit échelles ont été passées aux mêmes moments par les trois groupes (début janvier en pré-test, fin février en post-test), puis à nouveau deux mois plus tard par les groupes mindfulness et Tai Chi (suivi à deux mois, fin avril). Les stages ont été organisés et dirigés par chacun des deux auteurs de cet article, respectivement expérimentés en mindfulness et en Tai Chi Chuan.

\section{Méthode}

\section{Hypothèses}

Hypothèse 1. Les stages de pleine conscience et de Tai Chi Chuan diminuent les difficultés de santé mentale (santé générale, stress, anxiété, dépression) et favorisent la santé mentale positive (auto-efficacité, satisfaction de vie, bien-être et aptitude en mindfulness) des étudiants.

Hypothèse 2. Les bénéfices des stages sont corrélés au temps de pratique quotidienne effectuée à domicile pendant la durée du stage.

\section{Participants}

Quarante et un étudiants se sont préinscrits, 37 ont commencé leur stage (13 Tai Chi, 10 mindfulness, 14 témoins), 33 l'ont achevé en participant à toutes les séances ou aux séances de rattrapage proposées dans la semaine aux absents (maladies hivernales, intempéries, circonstances familiales), ont rendu tous les questionnaires et ont été inclus dans l'analyse (11 Tai Chi, 10 mindfulness et 12 témoins) (Tableau 1).

Les 33 étudiants sont issus de différentes composantes de l'université de Limoges (lettres, sciences humaines, IUFM, sciences, médecine, droit et sciences économiques, écoles d'ingénieur, IUT). En inscription libre, Tai Chi ou mindfulness dans la période de janvierfévrier (groupes expérimentaux) ou de mars-avril (groupe témoin en liste d'attente) (Tableau 2). Les stages ont duré huit semaines ( $18 \mathrm{~h}$, une séance de $2 \mathrm{~h} 15$ par semaine). Les périodes ont été choisies pour se dérouler sans coupure des vacances. Le groupe témoin comprend des étudiants en liste d'attente à la fois du stage Tai Chi et du stage mindfulness. 
La recherche a été organisée en 2012 à l'université de Limoges avec l'appui de l'institut universitaire de formation des maîtres (IUFM), du laboratoire FRED (EA 6311) de la faculté des lettres et des sciences humaines et du service universitaire des activités physiques et sportives (SUAPS). L'information a été diffusée par l'université de Limoges : mailing, web, radio, campagne d'affichage. L'inscription était gratuite. Conditions requises : ne pas être affecté de problèmes de santé importants; ne pas être sous suivi psychothérapeutique; s'efforcer ne pas manquer de séance (condition non impérative); se préparer à engager une pratique personnelle de 30 min de Tai Chi ou de mindfulness chez soi cinq jours sur sept pendant les huit semaines du stage (condition non impérative).

Tableau 1 Caractéristiques des participants.

Participant characteristics.

\begin{tabular}{|l|c|c|c|}
\hline & $\begin{array}{c}\text { Tai Chi } \\
\text { étudiants } \\
\mathrm{n}=11\end{array}$ & $\begin{array}{c}\text { Mindfulness } \\
\text { étudiants } \\
\mathrm{n}=10\end{array}$ & $\begin{array}{c}\text { Gr.témoin } \\
\text { étudiants } \\
\mathrm{n}=12\end{array}$ \\
\hline $\begin{array}{l}\text { Genre } \\
\text { Hommes } \\
\text { Femmes }\end{array}$ & 2 & 3 & 1 \\
\hline $\begin{array}{l}\text { Age } \\
\text { moyenne } \\
\text { (écart-type) }\end{array}$ & $\begin{array}{c}23,55 \\
(4,37)\end{array}$ & $\begin{array}{c}27,3 \\
(12,31)\end{array}$ & $\begin{array}{c}24,5 \\
(4,30)\end{array}$ \\
\hline
\end{tabular}

\section{Qualification des auteurs}

Le premier auteur pratique la méditation bouddhique depuis une vingtaine d'années dans la lignée tibétaine Kagyupa introduite en France par Lama Guendune Rinpoché. Il a suivi vingt semaines d'enseignement dispensés par Tarab Tulku Rinpoché (lignée Gelugpa) et Lene Handberg sur la philosophie bouddhique et les applications de la méditation en psychothérapie (Paris), des stages MBCT (Crêt-Bérard, Suisse), MBSR (ADM, France), des conférences de Kabat-Zinn, des retraites de pleine conscience au Village des Pruniers de Tich Nhat Hanh et dans des centres Kagyupa. Maître de conférences en psychologie, il intègre la thérapie basée sur la pleine conscience dans son activité clinique TCC.

De nationalité chinoise, la seconde auteure est $5^{\mathrm{e}}$ Duan des arts martiaux chinois, Tai Chi Chuan de style Chen traditionnel. Formée en Chine à Zheng Zhou par le Maître Zhang Dongwu. Elle a obtenu la première place à la Compétition internationale de Tai Ji Quan 2009 à Jiao Zuo, délivrée par l'administration générale du sport de Chine. Elle est enseignante vacataire de Tai Chi Chuan au service universitaire des activités physiques et sportives de l'université de Limoges. Doctorante, sa thèse porte sur les bénéfices que le Tai Chi Chuan procure aux étudiants en matière de réduction du stress et d'amélioration du bien-être.

\section{Instruments et modalités d'évaluation, traitement statistique}

Les données ont été recueillies à partir de huit échelles, d'un relevé de pratique et d'une question finale :

- quatre indicateurs ont été retenus pour mesurer les difficultés de santé mentale :

Le questionnaire général de santé GHQ-28 (symptômes somatiques, anxiété et insomnie, dysfonctionnement social, dépression), traduit en français et validé [92,93] : coté en échelle 
de Likert 0-1-2-3. L'échelle de stress perçu PSS-14 [94] traduite par Bruchon-Schweitzer et validée [95-96]. L'anxiété et la dépression mesurées par l'échelle HAD (HAD anxiété et HAD dépression) traduite par Lepine et al. et validée [97-99].

\section{- quatre indicateurs ont été choisis pour mesurer la santé mentale positive :}

L'échelle d'auto-efficacité de Schwarzer et Jerusalem (General Self-Efficacy Scale) adaptée en français et validée dans 23 pays [100-102] qui mesure le sentiment d'efficacité général perçu. L'échelle de satisfaction de vie SWLS, avec validation franco-canadienne [103]: habituellement utilisée comme mesure d'une composante du bien-être, elle complète l'échelle suivante WBQ. L'échelle de bien-être WBQ de Bradley [104] proposée en version française par Bruchon-Schweitzer en 12 items W-BQ12 [95]. L'échelle de mindfulness en 14 items FMI-14 [105] traduite et validée par Trousselard [106].

Exemples de questions : GHQ-28 : "vous êtes-vous senti(e) malade ? », " vous êtes-vous senti(e) constamment tendu(e) ou stressé(e) ?» (pas du tout, pas plus que d'habitude, un peu plus que d'habitude, bien plus que d'habitude), «avez-vous eu le sentiment que dans l'ensemble vous faisiez bien les choses ? » (mieux que d'habitude, aussi bien que d'habitude, moins bien que d'habitude, beaucoup moins bien que d'habitude), " avez-vous eu le sentiment que la vie ne vaut pas la peine d'être vécue?» (pas du tout, pas plus que d'habitude, un peu plus que d'habitude, bien plus que d'habitude). PSS-14: " avez-vous senti que les choses allaient comme vous le vouliez ? », " avez-vous été capable de contrôler la façon dont vous passiez votre temps ?» (jamais, presque jamais (rarement), parfois, assez souvent, très souvent). HAD : « je me fais souvent du soucis» (très souvent, assez souvent, occasionnellement, très occasionnellement), « je me sens heureux » (jamais, pas souvent, quelquefois, la plupart du temps). Sentiment d'auto-efficacité : "c'est facile pour moi de maintenir mon attention sur mes objectifs et accomplir mes buts », «je peux résoudre la plupart de mes problèmes si j'investis les efforts nécessaires » (pas du tout vrai, à peine vrai, moyennement vrai, totalement vrai). SWLS : « en général, ma vie correspond de près à mes idéaux », «mes conditions de vie sont excellentes » (fortement en désaccord, en désaccord, légèrement en désaccord, ni en désaccord ni en accord, légèrement en accord, en accord, fortement en accord). W-BQ12 : «je me sens triste et découragé(e) », « je me sens plein(e) d'énergie » (jamais ressenti, pas souvent ressenti, souvent ressenti, ressenti tout le temps).

L'échelle de mesure des aptitudes en mindfulness FMI-14 (Freiburg Mindfulness Inventory) a été conçue pour un public très large : méditants expérimentés sortant de longues retraites, méditants novices, population générale non méditante et enfin patients affectés de divers problèmes de santé, notamment psychologiques [105,106]. La version courte utilisée comprenant 14 questions est sémantiquement indépendante du contexte bouddhiste et de celui de la méditation. Nous avons donc pu la proposer aux trois groupes, d'autant plus que le Tai Chi Chuan est considéré comme une forme de "méditation en mouvement». Exemples de questions : «je suis réceptif(ve) à ce que je vis dans le moment présent », «je ressens mon corps dans les actes de la vie quotidienne (manger, cuisiner, parler, faire le ménage,...) », « je suis à l'écoute de mes sentiments sans me laisser déborder par eux » (presque jamais, occasionnellement, assez souvent, presque toujours). La limite principale du FMI-14, comme celle des autres échelles de mindfulness en auto-passation, tient à ce qu'elle ne mesure pas l'expérience réelle de mindfulness vécue par les sujets, mais ce qu'ils en rapportent de manière déclarative [7]. 
Les huit échelles ont été auto-administrées dans l'ordre de présentation ci-dessus. Elles comprennent en tout 97 questions. Leur passation s'est effectuée en trois temps M1, M2, M3, suivant le plan quasi expérimental du Tableau 2 :

- M1 : $1^{\text {re }}$ passation du questionnaire (Q1) à huit échelles au début de la $1^{\text {re }}$ séance du stage. Ànonymement, sur une durée libre (environ $20 \mathrm{mn}$ ). À la même date pour les deux groupes expérimentaux Tai Chi, mindfulness et le groupe témoin en liste d'attente ;

- M2 : $2^{\mathrm{e}}$ passation (Q2) à domicile quelques jours après la fin du stage, dans cette même période pour les trois groupes. Renvoyés anonymement par courrier postal ;

- M3 : $3^{\mathrm{e}}$ passation (Q3) à domicile, deux mois après la fin du stage. Groupes Tai Chi et mindfulness seulement. Retour anonyme par courrier postal.

- relevé quotidien du temps de pratique : il rapporte le temps d'exercice personnel à domicile de Tai Chi Chuan ou de "pratique formelle» de mindfulness pendant les huit semaines du stage (relevé anonyme). Le temps de "pratique informelle » de mindfulness n'a pas été comptabilisé car il nous paraît moins rigoureusement mesurable. Aucune consigne de poursuite des exercices après le stage n'a été donnée. Chacun était libre de faire à sa guise pendant les deux mois suivants, tout en sachant que le Q3 serait à remplir à l'issue de cette période.

- question finale : «à quel point le stage a été important pour vous ? (réponse anonyme, échelle 0/10).

L'analyse des résultats a été réalisée avec le logiciel XLSTAT 2012 : moyennes, écartstypes (non biaisés), Anova mixtes à 2 facteurs, test $t$ de Student pour échantillons appariés avec calcul des valeurs de $p$ pour les comparaisons analytiques pré-/post-/suivi à deux mois ou, dans quelques rares cas, test des rangs signés de Wilcoxon lorsque le $t$ de Student ne pouvait être appliqué. Les corrélations ont été calculées avec le coefficient $r$ de BravaisPearson.

Tableau 2 Plan quasi expérimental résumé.

Summary of the Experimental design.

\begin{tabular}{lcccc}
\hline $\begin{array}{l}\text { Groupes } \\
\text { expérimentaux }\end{array}$ & $\begin{array}{c}\text { Pré-test Q1 } \\
\text { début janvier }\end{array}$ & $\begin{array}{c}\text { Stage Tai Chi ou } \\
\text { IBPC janvier-février }\end{array}$ & $\begin{array}{c}\text { Post-test Q2 } \\
\text { fin février }\end{array}$ & $\begin{array}{c}\text { Suivi à 2 mois } \\
\text { test Q3 fin avril }\end{array}$ \\
$\begin{array}{l}\text { Groupe témoin } \\
\text { en liste d'attente }\end{array}$ & $\begin{array}{c}\text { Pré-test Q1 } \\
\text { début janvier }\end{array}$ & Pas de stage & $\begin{array}{c}\text { Post-test Q2 } \\
\text { fin février }\end{array}$ & $\begin{array}{c}\text { Stage Tai Chi ou } \\
\text { IBPC mars-avril, pas } \\
\text { de Q3 }\end{array}$ \\
\hline
\end{tabular}

IBPC : intervention basée sur la pleine conscience ; MBI : mindfulness based intervention

\section{Procédure et déroulement des séances}

Les séances de Tai Chi Chuan se sont déroulées dans le gymnase de l'IUFM. Elles ont été construites sur la base des cinq modalités suivantes :

- mouvements d'échauffement et d'assouplissement des articulations, relâchement des muscles, étirement des tendons ;

- entraînement de base ( $1^{\text {re }}$ partie) : Chan Si Jin (« enrouler la soie »). Synchronisation entre toutes les parties du corps par des mouvements en spirales ; 
- entraînement de base ( $2^{\mathrm{e}}$ partie) : les déplacements. Mouvements en déplacement engageant un bon équilibre du corps ;

- Zhan Zhuang (« la posture de l'arbre »). Posture destinée à renforcer les jambes, relâcher le corps, réguler la respiration et travailler la concentration ;

- apprentissage d'un petit enchaînement incluant quatre mouvements importants.

Le stage de pleine conscience s'est tenu dans la salle de musique de l'IUFM. Il était élaboré à partir du protocole MBSR de Kabat-Zinn [6] et MBCT de Segal, Williams, Teasdale [23] en huit séances :

- introduction à la pleine conscience ;

- stress et mindfulness-based stress reduction (MBSR);

- trois modes d'expérience : pensées, émotions, sensations corporelles ;

- rester présent ;

- exploration en pleine conscience de situations difficiles ;

- transformer la relation à nos jugements, nos interprétations des faits, nos pensées ;

- et passage à l'action et approfondissements.

Les séances comprenaient des apports théoriques, de nombreux exercices et des moments d'échange. La vie personnelle, privée n'était pas portée à la connaissance du groupe, les discussions restant centrées sur les exercices réalisés.

Invités à pratiquer en plus des séances de Tai Chi ou mindfulness 30 min d'exercices à domicile cinq jours sur sept pendant les huit semaines du stage, les étudiants devaient noter anonymement leur temps effectif de pratique quotidienne personnelle sur un calendrier qui leur était fourni. Le but poursuivi était à la fois de favoriser l'observance et d'examiner, comme dans les récentes recherches mentionnées plus haut, si les bénéfices des stages sont corrélés à ce temps de pratique.

\section{Résultats}

Les mesures des huit échelles sont réparties sur deux versants : les difficultés de santé mentale : (GHQ, PSS, HAD anxiété, HAD dépression) et la santé mentale positive :) (autoefficacité, satisfaction de vie SWLS, bien-être WBQ-12, aptitude en mindfulness FMI-14).

L'affectation des participants dans les groupes Tai Chi, mindfulness, témoin ayant relevé de leur libre choix, nous avons examiné l'homogénéité des trois groupes relativement aux scores obtenus sur chacune des huit échelles avant traitement. Les analyses de variance à un facteur (traitement Tai Chi, mindfulness, témoin) appliquées à la condition expérimentale " pré » sur les huit variables indépendantes QHQ, PSS, HADanx, HADdép, Auto-eff, SWLS, WBQ, FMI ne révèlent aucune différence significative au seuil de 0,05 entre les mesures relevées dans chaque groupe ( $p>0,05$ partout). Au regard de cette analyse les trois groupes se présentent comme homogènes au pré-test Q1. Cette équivalence globale sur chacune des huit échelles se conserve dans une comparaison des groupes deux à deux: Tai Chi/mindfulness, Tai Chi/témoin, mindfulness/témoin, en tout 24 comparaisons. Les Anova conduites par paires de groupes (ou tests $t$ de Student) ainsi que les tests de Fisher (LSD) et de Tukey (HSD) ne font pas apparaître de différence significative au seuil $p$ de 0,05 sur chaque échelle du pré-test. Des différences apparaissent cependant à un seuil moindre $(0,05<p<0,18)$, avec une valeur du $F$ relativement élevée $(F \geq 2)$, pour cinq de ces 24 
comparaisons : GHQ Tai Chi/mindfulness, HADanxiété Tai Chi/mindfulness, HADanxiété mindfulness/témoin, auto-efficacité Tai Chi/mindfulness, FMI mindfulness/témoin. Cette relative hétérogénéité se retrouve dans les valeurs des moyennes des groupes concernés, qui s'avèrent assez nettement différentes dans chacun des cinq cas : 31,18/23,70; 9,82/7,20 ; $7,20 / 10,17 ; 29,36 / 31,70 ; 39,30 / 35,67$ (Tableau 3). En outre, les âges moyens des groupes Tai Chi et mindfulness sont également assez différents : 23,5 ans et 27,3 ans (Tableau 1). Ces différences observées dans les niveaux de base des échelles et dans les âges moyens nous conduisent à considérer les groupes mindfulness et Tai Chi comme non équivalents.

Les scores des participants (sujets Sn) ont été recueillis sur chacune des huit échelles du questionnaire selon les deux plans $\mathrm{Sn}<\mathrm{T} 3>* \mathrm{M} 2$ et $\mathrm{Sn}<\mathrm{T} 2>* \mathrm{M} 3$. Le premier plan [3(Traitement) * 2(Moment : pré-test vs post-test)] correspond aux deux moments d'évaluation (début/fin de stage) des trois groupes (ou traitements) Tai Chi Chuan, mindfulness, témoin. Le second plan [2(Traitement: Tai Chi vs mindfulness) * 3(Moment: pré-test vs post-test vs post à deux mois)] correspond aux trois moments d'évaluation (début/fin de stage/suivi à deux mois) des deux groupes Tai Chi et mindfulness; le groupe témoin, en liste d'attente, n'a pas fait l'objet de suivi à deux mois (Tableau 2).

Les groupes mindfulness et Tai Chi étant considérés comme non équivalents, l'analyse statistique du plan $\mathrm{Sn}<\mathrm{T} 3>* \mathrm{M} 2$ a été décomposée en deux sous plans quasi expérimentaux de type $\mathrm{Sn}<\mathrm{T} 2\rangle^{*} \mathrm{M} 2$ sur lesquels ont été effectuées des Anova: $1^{\text {er }}$ sous plan Sn $<$ TTaiChi/témoin $>*$ M2 : [2(Traitement : Tai Chi vs témoin) *2(Moment : pré-test vs post-test)] $; 2^{\text {nd }}$ sous plan $\mathrm{Sn}<$ Tmindfulness/témoin $>* \mathrm{M} 2:$ [2(Traitement : mindfulness vs témoin) * 2(Moment : pré-test vs post-test)]. Pour le plan $\mathrm{Sn}<\mathrm{T} 2>* \mathrm{M} 3$, la non équivalence des groupes mindfulness et Tai Chi a conduit à réaliser des comparaisons indépendantes de ces deux groupes, les résultats obtenus aux trois moments d'évaluation étant analysés pour chaque groupe séparément au moyen de $t$ de Student. L'ensemble de l'analyse statistique a été réalisée sur les données brutes réelles, non tronquées, non winsorisées.

\section{Difficultés de santé mentale : échelles GHQ, PSS, HAD anxiété, HAD dépression}

\section{- GHQ-28}

Les analyses de variance mixte à deux facteurs $\mathrm{Sn}<\mathrm{TTaiChi} /$ témoin $>*$ M2 et Sn $<$ Tmindfulness/témoin $>*$ M2 ne montrent pas d'effet simple, principal, lié au traitement $\mathrm{T}$ (Tai Chi ou témoin et mindfulness ou témoin) sur les scores recueillis à l'échelle GHQ sur l'ensemble des deux moments M1 du pré-test Q1 et M2 du post-test Q2. Les scores sont statistiquement semblables à un seuil $p>0,05$. Ces résultats étaient plutôt attendus dans la mesure où l'homogénéité des groupes Tai Chi et témoin, leur équivalence globale observée, comme mentionné plus haut, avant traitement au pré-test $\mathrm{Q} 1(p=0,42)$, a pour conséquence d'atténuer l'effet principal du traitement sur la totalité des relevés [Q1, Q2] de GHQ, et de même pour les groupes mindfulness et témoin (homogènes, équivalents, au pré-test Q1 avec $p=0,44)$.

Nous observons en revanche un effet principal très significatif lié aux moments M1, M2 de passation du questionnaire sur l'échelle GHQ pour les deux sous plans $\mathrm{Sn}<$ TTaiChi/témoin $>* \mathrm{M} 2[\mathrm{~F}(1,21)=16,40 ; p<0,001]$ et $\mathrm{Sn}<$ Tmindfulness/témoin $>* \mathrm{M} 2$ $[\mathrm{F}(1,20)=8,57 ; p<0,006]$. Les scores recueillis sur la totalité des deux groupes Tai Chi et témoin ( $1^{\text {er }}$ sous plan), et des deux groupes mindfulness et témoin $\left(2^{\text {nd }}\right.$ sous plan), sont très significativement différents $(p<0,01)$ dans les mesures du pré-test Q1 comparativement à 
celles du post-test Q2. L'importance de cet effet est relativement surprenante sachant que le groupe témoin n'est pas supposé enregistrer de modifications notables entre M1 et M2 (mais cela s'avérera ne pas être le cas ici), ce qui devrait avoir pour conséquence d'atténuer tout différence générale liée au moment sur l'ensemble des relevés GHQ des deux groupes TaiChi/témoin et des deux groupes mindfulness/témoin. Le recours à des comparaisons analytiques entre les scores GHQ recueillis aux trois moments M1, M2, M3 dans chacun des trois groupes va nous permettre, plus loin, d'éclaircir cette question (Tableau 3).

Nous attendions un effet important d'interaction Traitement * Moment dans les deux sous plans $\mathrm{Sn}<$ TTaiChi/témoin $>* \mathrm{M} 2$ et $\mathrm{Sn}<$ Tmindfulness/témoin $>* \mathrm{M} 2$, traduisant respectivement les incidences différentes des traitements Tai Chi/témoin et mindfulness/témoin sur les scores atteints à l'échelle GHQ du post-test Q2 comparativement à ceux du pré-test Q1. En d'autres termes, la modalité du traitement suivi (Tai Chi ou témoin, mindfulness ou témoin) devrait avoir une influence significative sur l'amélioration des scores au GHQ entre leur valeur au moment du pré-test GHQ1 et leur valeur au moment du post-test GHQ2. Mais ce n'est pas le cas, il n'y a pas d'interaction dans le sous plan $\mathrm{Sn}<$ TTaiChi/témoin $>*$ M2 $[\mathrm{F}(1,21)=2,02$; NS], ni dans le sous plan Sn $<$ Tmindfulness/témoin $>*$ M2 $[\mathrm{F}(1,20)=0,17$; NS $]$.

Nous approfondissons l'analyse en procédant aux comparaisons analytiques présentées au Tableau 3. Pour faciliter la lecture des comparaisons, ce tableau rapporte les résultats sur les deux plans $\mathrm{Sn}<\mathrm{T} 3>* \mathrm{M} 2$ et $\mathrm{Sn}<\mathrm{T} 2>* \mathrm{M} 3$ : moyennes, écart-types et valeurs de $p$. Dans les quatre premières échelles de difficultés de santé mentale (:) santé générale GHQ, stress PSS, HAD anxiété, HAD dépression, l'état s'améliore lorsque les scores pré/post diminuent; dans les 4 dernières échelles de santé mentale positive (:) auto-efficacité, satisfaction de vie SWLS, bien-être WBQ, aptitude en mindfulness FMI, l'état s'améliore lorsque les scores pré/post augmentent. L'ensemble des comparaisons par paires (contrastes) a été réalisé avec le test $t$ de Student ou parfois avec le test des rangs signés de Wilcoxon lorsque la normalité des différences Q2-Q1 (pré-/post) ou Q3-Q1 (pré-/suivi à deux mois) n'était pas acquise. La normalité a été étudiée avec les quatre tests de Shapiro-Wilk, Anderson-Darling, Lilliefors, Jarque-Bera. Nous avons fait figurer en caractères gras dans des cases grisées les comparaisons révélant des différences très significatives (valeur de $p<0,01$ ), en cases simplement grisées les comparaisons significatives $(p<0,05)$ et en cases blanches les comparaisons non significatives (au seuil de 0,05). 
15

\begin{tabular}{|c|c|c|c|c|c|c|c|c|}
\hline 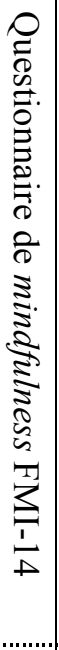 & 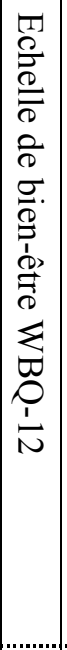 & 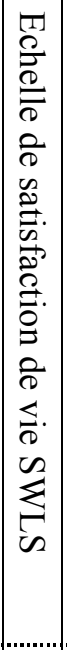 & 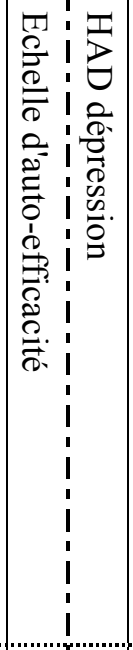 & 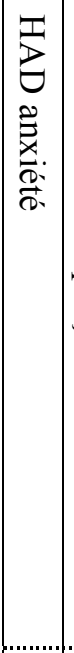 & 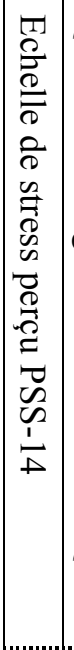 & 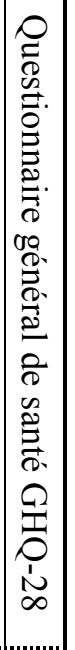 & 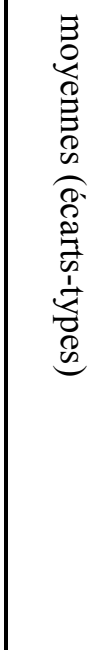 & 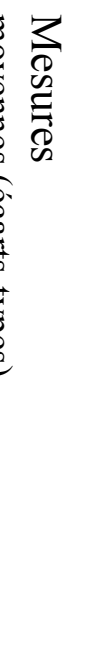 \\
\hline () & (C) & (:) & (:) ( & (:) & (:) & (:) & & \\
\hline 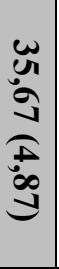 & $\begin{array}{l}\tilde{0} \\
0 \\
u \\
u \\
0 \\
0 \\
0\end{array}$ & 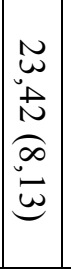 & 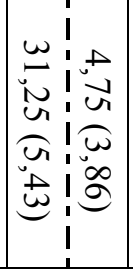 & $\mid \begin{array}{l}\overrightarrow{0} \\
\vdots \\
v \\
\omega \\
\omega \\
ن \\
0\end{array}$ & 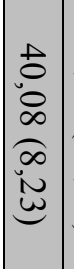 & 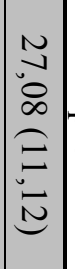 & : & 2 \\
\hline 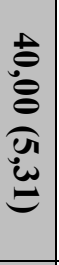 & $\begin{array}{l}N \\
N \\
\pm \\
\pm \\
U \\
N \\
N\end{array}$ & 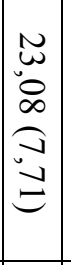 & 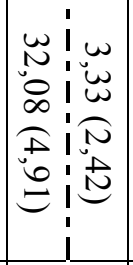 & 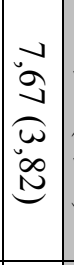 & 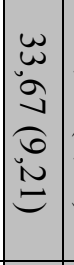 & 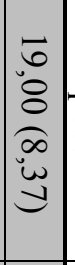 & 兽 & 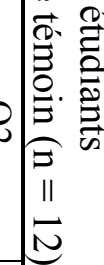 \\
\hline$\stackrel{5}{5}$ & $\underset{\infty}{\stackrel{0}{a}}$ & $\stackrel{0}{v}$ & $\begin{array}{l:l}0 & 0 \\
0 & 8 \\
心 & 心 \\
\sim & *\end{array}$ & $\begin{array}{l}\stackrel{0}{0} \\
\stackrel{1}{1}\end{array}$ & $\begin{array}{l}0 \\
0 \\
0 \\
心\end{array}$ & $\begin{array}{l}0 \\
0 \\
+\end{array}$ & $\frac{\sqrt{2}}{10}$ & \\
\hline
\end{tabular}

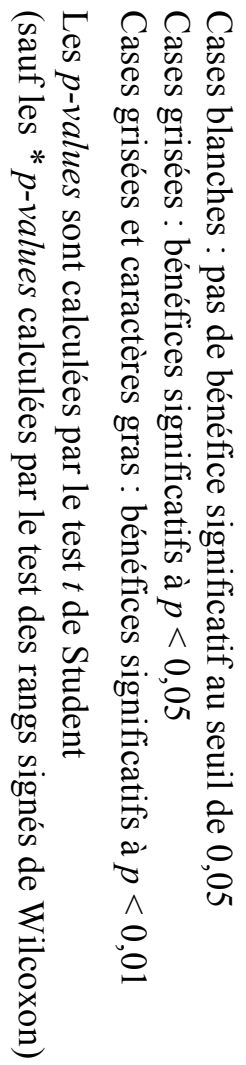

\begin{tabular}{|c|c|c|c|c|c|c|c|c|}
\hline 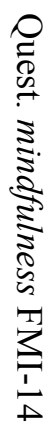 & 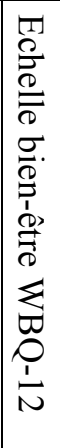 & 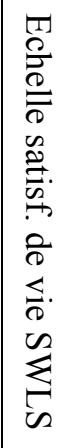 & 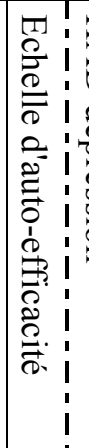 & 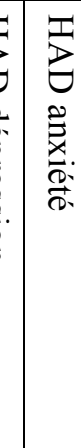 & $\begin{array}{l}1 \\
0 \\
0 \\
0 \\
0 \\
0 \\
0 \\
0 \\
0 \\
0 \\
0 \\
0 \\
0 \\
0 \\
0 \\
0 \\
0 \\
0 \\
0 \\
1 \\
1 \\
\end{array}$ & 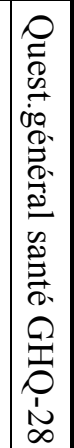 & $\begin{array}{l}\equiv \\
0 \\
0 \\
0 \\
0 \\
0 \\
0 \\
0 \\
8 \\
0 \\
0 \\
1 \\
1 \\
0 \\
0 \\
0\end{array}$ & 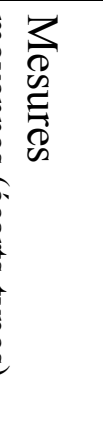 \\
\hline & 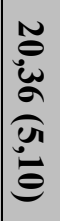 & 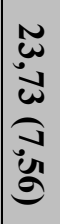 & 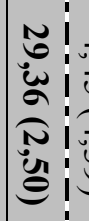 & $\begin{array}{l}0 \\
\infty \\
N \\
\stackrel{\infty}{+} \\
\stackrel{N}{N}\end{array}$ & $\begin{array}{l} \pm \\
\vdots \\
0 \\
0 \\
0 \\
0 \\
0\end{array}$ & 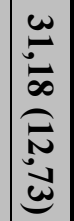 & 蛋. & $\frac{N}{N}$ \\
\hline & 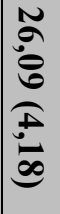 & 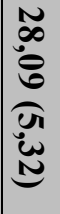 & 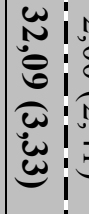 & 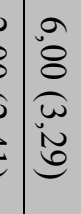 & 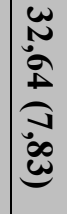 & $\left|\begin{array}{c}\vec{F} \\
\dot{\omega} \\
\sigma \\
\hat{\sigma} \\
\ddot{a} \\
\overrightarrow{0}\end{array}\right|$ & 要 & 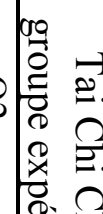 \\
\hline & $\stackrel{\leftrightarrow}{8}$ & $\ddot{8}$ & $\therefore$ & $\begin{array}{l}0 \\
\text { in } \\
u\end{array}$ & $\stackrel{\vec{s}}{\stackrel{s}{s}}$ & $\stackrel{\infty}{\infty}$ & 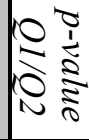 & 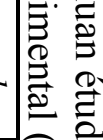 \\
\hline \begin{tabular}{l}
$\omega$ \\
0 \\
0 \\
\multirow{\sigma}{*}{}
\end{tabular} & 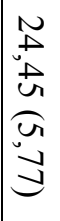 & 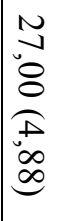 & $\begin{array}{l}\omega \\
\omega \\
\omega \\
\omega \\
\tilde{I} \\
\omega \\
\omega\end{array}$ & 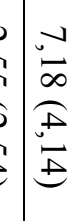 & \begin{tabular}{l}
$\omega$ \\
$\omega$ \\
$w$ \\
$\omega$ \\
0 \\
0 \\
\multirow{0}{0}{}
\end{tabular} & $\begin{array}{c}\vec{u} \\
\infty \\
\infty \\
N \\
0 \\
\infty \\
\infty \\
0\end{array}$ & 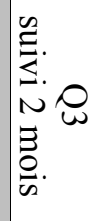 & E \\
\hline 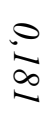 & $\begin{array}{l}0 \\
8 \\
8\end{array}$ & $\begin{array}{l}0 \\
0 \\
ن \\
u\end{array}$ & $\begin{array}{l}01 \\
0 \\
0\end{array}$ & \begin{tabular}{l}
$i$ \\
\multirow{N}{*}{} \\
$*$
\end{tabular} & $\begin{array}{l}8 \\
8 \\
0\end{array}$ & $\begin{array}{l}\dot{0} \\
\tilde{0} \\
a\end{array}$ & 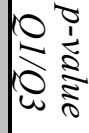 & \\
\hline
\end{tabular}

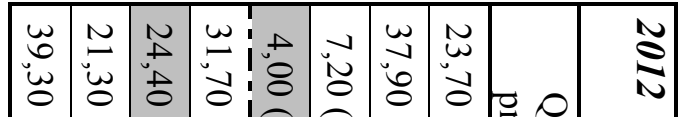

OU

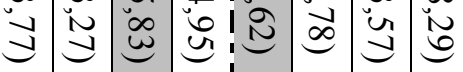

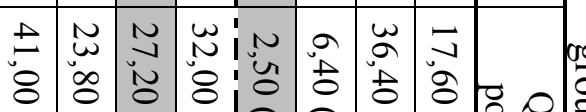

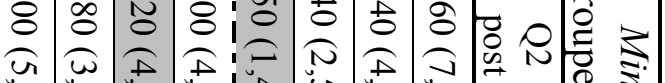
司

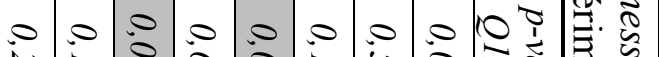

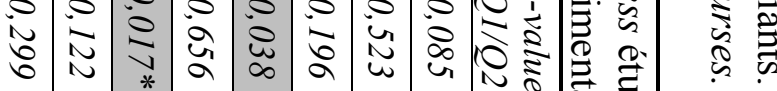

\begin{tabular}{|c|c|c|c|c|c|c|c|}
\hline 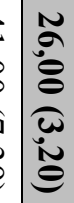 & 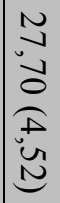 & $\begin{array}{l}w \\
w \\
\dot{0} \\
0 \\
\hat{0} \\
\ddot{0} \\
0\end{array}$ & $\begin{array}{l}n \\
\tilde{w} \\
0 \\
O \\
0 \\
\infty \\
0 \\
0\end{array}$ & 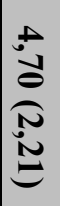 & 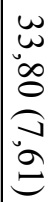 & $\begin{array}{l}\overrightarrow{0} \\
\dot{\omega} \\
0 \\
0 \\
0 \\
\omega \\
\omega\end{array}$ & 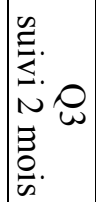 \\
\hline 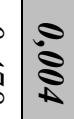 & $\begin{array}{l}\dot{B} \\
\dot{E}\end{array}$ & $\begin{array}{l}0 \\
\tilde{N} \\
* \\
*\end{array}$ & $\begin{array}{l}\stackrel{0}{0} \\
\stackrel{0}{\sim}\end{array}$ & $\ddot{8}$ & $\stackrel{0}{i}$ & 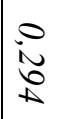 & 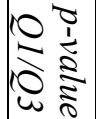 \\
\hline
\end{tabular}

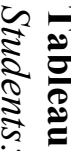

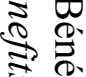

จิ

कृ

iे

논

疍

s.

Sin

$\sum^{2} \overline{0}$

है

$\overrightarrow{0}$

今.

官

告

范

เ के

$\rightarrow$ ․․․

2.

$\Omega E$.

§

\$

इิ คे

공

ㄴ.

롱.

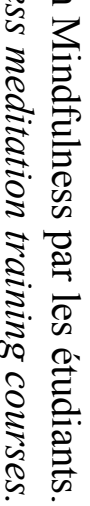

o 
Sur l'échelle GHQ des difficultés de santé générale, nous découvrons d'abord une amélioration significative du groupe témoin $(p=0,044)$. Nous pouvons vraisemblablement attribuer cette performance a priori surprenante au fait que le questionnaire Q1 de début janvier a été passé pendant la semaine tendue des examens partiels, tandis que le Q2 de fin février en était dégagé. Le calendrier de l'année universitaire ne permettait pas d'introduire huit semaines consécutives de stage non interrompues par des vacances, prolongées de huit autres semaines de suivi à deux mois, sans que le passage de l'un des trois questionnaires (Q1, Q2, Q3) ne survienne dans une période d'examen. L'ensemble des étudiants a souligné le poids de ce facteur de stress des examens, qui joue un rôle important dans la fragilité de cette population. Certaines recherches ayant pour objet des interventions sur des groupes d'étudiants prennent également en compte cette variabilité de leur état de santé, en ou hors période d'examen, dans l'interprétation des résultats [18].

L'amélioration du groupe témoin explique certainement l'effet principal très significatif lié aux moments M1, M2 relevé plus haut dans les Anova globales des deux sous plans $\mathrm{Sn}<$ TTaiChi/témoin $>* \mathrm{M} 2$ et $\mathrm{Sn}<$ Tmindfulness/témoin $>* \mathrm{M} 2$, de même que l'absence d'interaction Traitement * Moment dans ces deux sous plans. Nous observons ensuite une amélioration pré-/post-traitement très significative $(p=0,008)$ du groupe Tai Chi. En revanche, l'amélioration moyenne relevée dans le groupe mindfulness ne se révèle pas significative mais seulement tendancielle $(p=0,085)$. Le bénéfice du groupe Tai Chi se maintient sur le suivi à deux mois GHQ3 mais avec une significativité moins importante $(p=0,026)$ que pour le post-test GHQ2 $(p=0,008)$.

\section{- PSS-14}

Assez semblablement à l'échelle GHQ :

- nous ne rencontrons pas d'effet principal lié au traitement $\mathrm{T}$ (Tai Chi ou témoin et mindfulness ou témoin) sur les scores recueillis à l'échelle de stress PSS, sur aucun des deux sous plans $\mathrm{Sn}<$ TTaiChi/témoin $>*$ M2 et $\mathrm{Sn}<$ Tmindfulness/témoin $>*$ M2 ;

- nous observons un effet principal très significatif du moment M1, M2 de passation de l'échelle PSS pour le sous plan $\mathrm{Sn}<$ TTaiChi/témoin $>* \mathrm{M} 2[\mathrm{~F}(1,21)=20,39 ; p<0,001]$ et significatif pour le sous plan $\mathrm{Sn}<\mathrm{T}$ mindfulness/témoin $>* \mathrm{M} 2[\mathrm{~F}(1,20)=5,56 ; p<0,023]$;

- nous n'obtenons pas d'effet d'interaction Traitement * Moment dans le sous plan $\mathrm{Sn}<$ TTaiChi/témoin $>* \mathrm{M} 2 \quad[\mathrm{~F}(1,21)=0,35 ; \mathrm{NS}]$, ni dans le sous plan $\mathrm{Sn}<$ Tmindfulness/témoin $>* \mathrm{M} 2[\mathrm{~F}(1,20)=2,14 ; \mathrm{NS}]$.

Les comparaisons analytiques entreprises conduisent aussi exactement aux mêmes résultats que pour l'échelle GHQ (Tableau 3) : une réduction significative du stress du groupe témoin $(p=0,022)$, une réduction plus importante, très significative, pour le groupe Tai Chi $(p=0,003)$ et en revanche, contrairement à notre attente de l'Hypothèse 1, pas d'amélioration pour le groupe mindfulness $(p=0,52)$. La réduction du stress du groupe Tai Chi se maintient sur le suivi à deux mois PSS3 de manière significative $(p=0,045)$.

\section{- HAD anxiété}

De la même manière que pour l'échelle PSS : il n'apparaît pas d'effet simple du traitement $\mathrm{T}$ sur aucun des deux sous plans $\mathrm{Sn}<$ TTaiChi/témoin $>*$ M2 et $\mathrm{Sn}<\mathrm{Tmindful}$ ness/témoin $>* \mathrm{M} 2$; il ressort un effet principal très significatif du moment $\mathrm{M}$ pour le sous plan $\mathrm{Sn}<$ TTaiChi/témoin $>* \mathrm{M} 2[\mathrm{~F}(1,21)=11,02 ; p<0,002]$ et significatif pour le sous plan $\mathrm{Sn}<$ Tmindfulness/témoin $>* \mathrm{M} 2[\mathrm{~F}(1,20)=5,02 ; p<0,031]$; il ne se produit pas d'effet d'in- 
teraction Traitement * Moment dans le sous plan $\mathrm{Sn}<\mathrm{TTaiChi} /$ témoin $>* \mathrm{M} 2[\mathrm{~F}(1,21)=0,48$; $\mathrm{NS}]$, ni dans le sous plan $\mathrm{Sn}<\mathrm{Tmindfulness/témoin}>* \mathrm{M} 2[\mathrm{~F}(1,20)=1,33 ; \mathrm{NS}]$.

Les comparaisons analytiques montrent une diminution significative de l'anxiété dans le groupe Tai Chi en pré-/post-test $(p=0,025)$, mais qui ne se maintient pas significativement dans le suivi à deux mois. Les deux autres groupes témoin et mindfulness n'enregistrent pas de bénéfices significatifs (à $p<0,05$ ) entre les deux moments M1 et M2. Une réduction de l'anxiété se manifeste seulement en M3 dans le groupe mindfulness $(p=0,005)$; on retrouve un résultat semblable dans d'autres recherches, comme celle de Ruths et al. [45] où la réduction de l'anxiété s'observe, non pas à la fin du stage MBCT (en post-test), mais dans le suivi à trois mois sur la base d'une corrélation entre le temps personnel de pratique quotidienne jusqu'à ces trois mois et la diminution du score d'anxiété-trait sur une échelle STAI.

\section{- HAD dépression}

Les résultats des Anova globales sont semblables à ceux rencontrés sur l'échelle GHQ : pas d'effet simple $d u$ facteur traitement $T$ sur aucun des deux sous plans $\mathrm{Sn}<\mathrm{TTaiChi} /$ témoin $>* \mathrm{M} 2$ et $\mathrm{Sn}<\mathrm{Tmindfulness/témoin}>* \mathrm{M} 2$; effet simple très significatif du facteur moment $\mathrm{M}$ pour les deux sous plans $\mathrm{Sn}<\mathrm{TTaiChi} /$ témoin $>* \mathrm{M} 2[\mathrm{~F}(1,21)=14,85$; $p<0,001]$ et $\mathrm{Sn}<\mathrm{T}$ mindfulness/témoin $>* \mathrm{M} 2[\mathrm{~F}(1,20)=11,13 ; p<0,002]$; pas d'effet d'interaction Traitement * Moment dans le sous plan $\mathrm{Sn}<$ TTaiChi/témoin $>* \mathrm{M} 2[\mathrm{~F}(1,21)=1,07$; $\mathrm{NS}]$, ni dans le sous plan $\mathrm{Sn}<\mathrm{T}$ mindfulness/témoin $>* \mathrm{M} 2[\mathrm{~F}(1,20)=0,01 ; \mathrm{NS}]$.

Les comparaisons analytiques font apparaître une diminution significative de la dépression en pré-/post-test dans le groupe Tai Chi $(p=0,015)$ et dans le groupe mindfulness $(p=0,038)$, mais qui ne se maintiennent pas significativement dans le suivi à deux mois. Le groupe témoin n'est pas loin d'enregistrer une réduction significative $(p=0,053)$.

\section{Santé mentale positive : échelles d'auto-efficacité, de satisfaction de vie SWLS, de bien-être WBQ-12, de mindfulness FMI-14}

\section{- Auto-efficacité}

Il n'apparait pas d'effet simple du facteur traitement $T$ sur aucun des deux sous plans $\mathrm{Sn}<$ TTaiChi/témoin $>* \mathrm{M} 2$ et $\mathrm{Sn}<$ Tmindfulness/témoin $>* \mathrm{M} 2$; il ressort un effet principal très significatif du moment M1, M2 de passation de l'échelle pour le sous plan $\mathrm{Sn}<$ TTaiChi/témoin $>* \mathrm{M} 2[\mathrm{~F}(1,21)=8,79 ; p<0,005]$, mais il ne se présente pas d'effet significatif pour le sous plan $\mathrm{Sn}<$ Tmindfulness/témoin $>* \mathrm{M} 2[\mathrm{~F}(1,20)=0,80 ; p<0,377]$; nous ne relevons pas d'effet d'interaction Traitement * Moment dans le sous plan $\mathrm{Sn}<$ TTaiChi/témoin $>*$ M2 $\quad[\mathrm{F}(1,21)=2,49 ; \mathrm{NS}], \quad$ ni dans le sous plan $\mathrm{Sn}<$ Tmindfulness/témoin $>* \mathrm{M} 2[\mathrm{~F}(1,20)=0,18 ; \mathrm{NS}]$.

Les comparaisons analytiques (ou contrastes) révèlent une augmentation très significative du sentiment d'auto-efficacité dans le groupe Tai Chi $(p=0,001)$, mais pas d'amélioration dans le groupe mindfulness ni dans le groupe témoin. L'amélioration du groupe Tai Chi se maintient significativement sur le suivi à deux mois $(p=0,029)$. Cet effet positif de la pratique du Tai Chi sur le sentiment d'efficacité général a déjà été signalé dans la littérature scientifique [71].

\section{- Satisfaction de vie SWLS}

Comme pour l'échelle précédente d'auto-efficacité : 
- il n'apparaît pas d'effet simple du traitement $\mathrm{T}$ sur aucun des deux sous plans $\mathrm{Sn}<$ TTaiChi/témoin $>*$ M2 et $\mathrm{Sn}<$ Tmindfulness/témoin $>* \mathrm{M} 2$;

- nous notons un effet principal très significatif du moment M1, M2 de passation de l'échelle pour le sous plan $\mathrm{Sn}<$ TTaiChi/témoin $>* \mathrm{M} 2[\mathrm{~F}(1,21)=7,55 ; p<0,009]$, mais nous ne rencontrons pas d'effet significatif pour le sous plan $\mathrm{Sn}<$ Tmindfulness/témoin $>* \mathrm{M} 2$ $[\mathrm{F}(1,20)=2,40 ; p<0,129]$.

À la différence des cinq échelles précédentes, nous relevons un effet d'interaction Traitement * Moment très significatif dans le sous plan $\mathrm{Sn}<\mathrm{TTaiChi} /$ témoin $>*$ M2 $[\mathrm{F}(1,21)=10,26 ; p<0,003]$, et tendanciel dans le sous plan $\mathrm{Sn}<\mathrm{Tmindfulness/témoin}>* \mathrm{M} 2$ $[\mathrm{F}(1,20)=3,88 ; p<0,056]$.

Les comparaisons analytiques confirment l'effet d'interaction Traitement * Moment, l'hétérogénéité des variations pré-/post-test SWLS enregistrées dans les deux sous plans : le sentiment de satisfaction de vie s'accroît très significativement dans le groupe Tai Chi $(p=0,001)$, significativement dans le groupe mindfulness $(p=0,017)$, alors que le groupe témoin n'obtient pas d'augmentation significative $(p=0,76)$. Les bénéfices acquis par le groupe Tai Chi se conservent tendanciellement sur le suivi à deux mois $(p=0,055)$, ils se conservent significativement pour le groupe mindfulness $(p=0,047)$.

\section{- Bien-être WBQ-12}

De même que pour les échelles PSS et HAD anxiété :

- il ne se dégage pas d'effet principal du traitement $\mathrm{T}$ sur aucun des deux sous plans $\mathrm{Sn}<$ TTaiChi/témoin $>*$ M2 et Sn $<$ Tmindfulness/témoin $>*$ M2 ;

- nous obtenons un effet principal très significatif du moment $M$ pour le sous plan $\mathrm{Sn}<$ TTaiChi/témoin $>* \mathrm{M} 2[\mathrm{~F}(1,21)=15,51 ; p<0,001]$ et significatif pour le sous plan $\mathrm{Sn}<$ Tmindfulness/témoin $>* \mathrm{M} 2[\mathrm{~F}(1,20)=5,13 ; p<0,029]$;

- en revanche, nous rencontrons un effet d'interaction Traitement * Moment significatif dans le sous plan $\mathrm{Sn}<$ TTaiChi/témoin $>* \mathrm{M} 2[\mathrm{~F}(1,21)=4,68 ; p<0,037]$, mais pas dans le sous plan $\mathrm{Sn}<$ Tmindfulness/témoin $>* \mathrm{M} 2[\mathrm{~F}(1,20)=0,21 ; \mathrm{NS}]$.

Les comparaisons analytiques révèlent une amplification très significative du sentiment de bien-être dans le groupe Tai Chi $(p=0,003)$, mais pas d'amélioration dans le groupe mindfulness ni dans le groupe témoin. L'amélioration du groupe Tai Chi ne se maintient pas sur le suivi à deux mois M3. Une augmentation du bien-être se manifeste seulement en M3 dans le groupe mindfulness $(p=0,004)$; Ruths et al. [45] observent, de leur côté, une augmentation du bien-être à la fin du stage MBCT («psychological well-being » mesuré sur une échelle GHQ-12), qui se conserve dans le suivi à trois mois sous la condition de l'observance d'une pratique quotidienne personnelle jusqu'à ces trois mois, en corrélation avec le temps de cette pratique.

\section{- Questionnaire de mindfulness FMI-14}

Semblablement aux échelles GHQ et HAD dépression :

- nous n'observons pas d'effet principal du traitement $\mathrm{T}$ sur aucun des deux sous plans $\mathrm{Sn}<$ TTaiChi/témoin $>*$ M2 et Sn $<$ Tmindfulness/témoin $>*$ M2 ;

- nous relevons un effet simple très significatif du facteur moment $\mathrm{M}$ pour les deux sous plans $\mathrm{Sn}<$ TTaiChi/témoin $>* \mathrm{M} 2[\mathrm{~F}(1,21)=42,41 ; p<0,001]$ et $\mathrm{Sn}<$ Tmindfulness/témoin $>* \mathrm{M} 2$ $[\mathrm{F}(1,20)=11,72 ; p<0,002]$; 
- pas d'effet d'interaction Traitement * Moment dans le sous plan $\mathrm{Sn}<$ TTaiChi/témoin $>$ * M2 $[\mathrm{F}(1,21)=0,48 ; \mathrm{NS}]$, ni dans le sous plan $\mathrm{Sn}<\mathrm{T}$ mindfulness/témoin $>* \mathrm{M} 2[\mathrm{~F}(1,20)=2,23$; NS].

Les comparaisons analytiques mettent en relief un progrès très significatif $(p=0,003)$ de l'aptitude en mindfulness des étudiants Tai Chi entre les moments M1 du pré-test et M2 du post-test, mais qui ne se maintient pas sur le suivi à deux mois M3. Contrairement à notre attente, aucun progrès significatif n'est rencontré chez les étudiants mindfulness $(p=0,299)$. Résultat surprenant, les étudiants du groupe témoin enregistrent au M2 de fin février un gain de mindfulness hautement significatif $(p=0,001)$ alors qu'ils n'ont pas encore fait leur stage de mars-avril ; ce résultat renforce notre interprétation selon laquelle le questionnaire Q2 de fin février a été rempli dans une meilleure condition générale d'équilibre et d'apaisement, permettant une certaine ouverture et réceptivité aux états intérieurs physiques et mentaux; alors que le Q1 de début janvier a été passé en pleine période d'examen, dans des conditions difficiles de tension et de stress générant une rétraction de la sensibilité aux états intérieurs, une crispation des relations corps/esprit, une diminution des facultés d'auto-observation.

\section{Corrélation des bénéfices $\mathrm{Q1/Q2}$ avec le temps de pratique quotidienne}

Avec le Tableau 4 nous constatons une assez forte observance de la consigne de pratique personnelle à domicile (" pratique formelle ») cinq jours sur sept durant 30 min pendant les huit semaines du stage. En moyenne quotidienne, calculée sur cinq jours/semaine : m (Tai Chi $)=15 \mathrm{~min} ; \mathrm{m}($ mindfulness $)=23,4 \mathrm{~min}$.

\section{Tableau 4}

Temps quotidien de pratique. Daily practising time

Corrélation avec les bénéfices obtenus. Correlation with the benefits achieved

Importance accordée au stage. Emphasis placed on the course

Temps moyen t de pratique/jour durant les 8 semaines du stage (écart-type)

\begin{tabular}{|c|c|}
\hline $\begin{array}{c}\text { Étudiants } \\
\text { Tai Chi }\end{array}$ & $\begin{array}{c}\text { Étudiants } \\
\text { mindfulness }\end{array}$ \\
\hline $15 \mathrm{~min}$ & $23,4 \mathrm{~min}$ \\
$(6,5 \mathrm{~min})$ & $(5,6 \mathrm{~min})$ \\
\hline
\end{tabular}

\begin{tabular}{|l|c|c|}
\hline $\begin{array}{c}\text { Corrélation entre t et les valeurs pré/post }(\Delta=\mathbf{Q} 2-\mathbf{Q} 1) \text { des huit échelles } \\
\text { coefficient } \boldsymbol{r} \text { de Pearson (valeur de p) }\end{array}$ & $\begin{array}{c}\text { étudiants } \\
\text { Tai Chi }\end{array}$ & $\begin{array}{c}\text { étudiants } \\
\text { mindfulness }\end{array}$ \\
\hline$\Delta$ Questionnaire général de santé GHQ-28 & NS & $-0,56(0,09)$ \\
\hline$\Delta$ Echelle de stress perçu PSS-14 & NS & $\mathbf{- 0 , 7 6 ( 0 , 0 1 )}$ \\
\hline$\Delta$ HAD anxiété & NS & $-0,62(0,06)$ \\
\hline$\Delta$ HAD dépression & NS & NS \\
\hline$\Delta$ Echelle d'auto-efficacité & NS & NS \\
\hline$\Delta$ Echelle de satisfaction de vie SWLS & NS & NS \\
\hline$\Delta$ Echelle de bien-être WBQ-12 & NS & NS \\
\hline$\Delta$ Questionnaire mindfulness FMI-14 & NS & $\mathbf{0 , 7 6 ( 0 , 0 1 )}$ \\
\hline$N S:$ non significatif $(r<0,5 ; p>0,05)$ & &
\end{tabular}

\begin{tabular}{|c|c|c|}
\hline $\begin{array}{c}\text { Question générale finale(anonyme): } \\
\text { à quel point le stage a été important pour vous ? }\end{array}$ & $\begin{array}{c}\text { étudiants } \\
\text { Tai Chi }\end{array}$ & $\begin{array}{c}\text { étudiants } \\
\text { mindfulness }\end{array}$ \\
\hline Importance moyenne accordée (0/10) (écart-type) & $8,95(0,93)$ & $5,67(1,90)$ \\
\hline
\end{tabular}

Les bénéfices pré-/post-traitement (Q1/Q2) relevés à l'issue des stages de Tai Chi et de mindfulness sont-ils corrélés avec ce temps d'exercice quotidien ? Chez les étudiants Tai Chi 
il ne ressort pas de corrélation. Chez les étudiants mindfulness nous découvrons une faible corrélation entre leur temps de pratique quotidienne et l'amélioration de leur santé générale GHQ $(r=0,558$ et $p<0,1)$, une corrélation moyenne avec la réduction de leur anxiété HAD ( $r=0,619$ et $p=0,056)$ et des corrélations particulièrement fortes avec la réduction de leur stress PSS $(r=0,759$ et $p=0,011)$ et le développement de leur aptitude en mindfulness FMI $(r=0,759$ et $p=0,006)$.

\section{Discussion}

Nous avons constaté une différence très importante des scores aux huit échelles entre les mesures pré-test Q1 de début janvier et post-test Q2 de fin février dans les trois groupes Tai Chi, mindfulness, témoin, découvrant ainsi une amélioration surprenante du groupe témoin. La période d'examen de début janvier semble avoir assez fortement affecté la santé et le stress des étudiants, impactant les scores du questionnaire Q1. Cette observation de la détérioration de la santé des étudiants en période d'examen est mentionnée dans d'autres recherches [107], et reflète peut-être aussi la vulnérabilité et la précarité de la condition étudiante mises en relief par les enquêtes épidémiologiques $[1,2]$.

Après ce premier constat, les résultats généraux de notre recherche font apparaître, dans les limites de notre expérience et sur la base des analyses de variance et des comparaisons analytiques, des effets positifs de la pratique du Tai Chi Chuan dans l'ensemble du groupe sur les deux versants de la réduction des difficultés de santé mentale $*$ (santé générale, stress, anxiété, dépression) et de l'amélioration de la santé mentale positive :) (auto-efficacité, satisfaction de vie, bien-être, aptitude en mindfulness) ; comparativement au groupe témoin.

Contrairement à notre attente de l'Hypothèse 1, la pratique de la pleine conscience ne conduit pas à des bénéfices significatifs pré-/post-traitement pour l'ensemble du groupe mindfulness, sauf dans le suivi à deux mois sur l'anxiété et le bien-être. Elle en procure en revanche individuellement, en corrélation avec le temps de pratique personnelle à domicile, principalement sur les plans de la réduction du stress, de l'anxiété et du développement de l'aptitude en mindfulness (Tableau 4). Ceci valide partiellement notre Hypothèse 2, et seulement pour ce groupe des étudiants mindfulness, car nous n'obtenons pas de corrélation entre le temps de pratique personnelle du Tai Chi et les bénéfices pré-/post-traitement enregistrés par les étudiants de ce groupe sur aucune des huit échelles.

Dans le champ des recherches sur les MBIs, certaines études ne constatent pas de relation entre la quantité de pratique personnelle quotidienne et les bénéfices recueillis, d'autres par contre en découvrent. Face à cette ambiguïté il est envisagé que les effets ne puissent apparaître qu'à partir d'un seuil critique de temps de pratique quotidienne $[45,46]$. Ainsi Carson et al. observent-ils des effets significatifs de la MBSR sur des participants exerçant en moyenne 32 min par jour de "pratique formelle», six jours par semaine pendant les huit semaines du stage [108]. Peut-être que le temps moyen de pratique quotidienne de mindfulness relevé dans notre stage $(23,4 \mathrm{~min} / \mathrm{j}, 5 \mathrm{j} / \mathrm{semaine}, 8$ semaines $)$ n'atteint-il pas ce seuil critique, ce qui expliquerait que des bénéfices n'apparaissent pas en fin de stage pour la totalité du groupe mais seulement individuellement pour ceux qui ont le plus pratiqué et qui, peut-être, auraient continué à pratiquer dans le suivi à deux mois ?

Nous pouvons supposer que l'assez forte observance relevée pour la pratique quotidienne du Tai Chi (15 min/j, $5 \mathrm{j} / \mathrm{semaine}, 8$ semaines $)$ et de la mindfulness $(23,4 \mathrm{~min} / \mathrm{j}, 5 \mathrm{j} / \mathrm{semaine}, 8$ 
semaines) témoigne de l'investissement et de l'attente des participants à l'égard de leur stage (Tableau 4). L'importance de cette motivation se retrouve dans le score attribué à la «question générale finale : à quel point le stage a été important pour vous? " à laquelle chacun a été convié à répondre anonymement par écrit à la fin du stage (Tableau 4). Sur une échelle 0/10, en moyenne : $\mathrm{m}$ (Tai Chi) $=8,95(0,93) ; \mathrm{m}($ mindfulness $)=5,67(1,90)$.

Les étudiants ont largement plébiscité le stage de Tai Chi, tandis que le stage mindfulness a été plus variablement investi (écart-type de 1,90 deux fois supérieur au 0,93 du groupe Tai Chi). De cet intérêt plus fort pour la pratique du Tai Chi, peut-on inférer l'hypothèse que le Tai Chi, de nature plus dynamique, forme de "méditation en mouvement», convient davantage aux jeunes et leur procure des bienfaits significatifs, alors que la méditation de pleine conscience, plus posée, profiterait davantage aux personnes plus mûres? Au demeurant, les étudiants du groupe mindfulness qui ont engagé une pratique personnelle quotidienne importante paraissent avoir eux aussi acquis des bénéfices significatifs (au regard des études de corrélation, Tableau 4).

Bien que les résultats de cette étude pilote soient encourageants, des limites incitent à la prudence. La taille totale de notre échantillon est réduite. Le protocole de recherche est quasi expérimental: contrôlé mais non randomisé. Les échantillons des groupes de pleine conscience et de Tai Chi Chuan sont considérés comme non équivalents. La superposition du moment de passation de l'un des trois questionnaires (le Q1) à une période d'examens n'a pu être évitée, la recherche étant conduite hors période de vacances sur plusieurs mois. Dans le contexte ouvert, chaleureux d'accueil des participants volontaires, l'implication des stagiaires a été importante. Une partie des résultats acquis peut être liée à ces simples conditions de vécu positif et nourrissant des groupes. Les questionnaires ont été remplis sur papier en autopassation anonyme, ce qui induit des biais de subjectivité dans le choix des réponses: mauvaise interprétation des questions, déni, exagération, minimisation, désir de plaire... Le suivi à distance n'a porté que sur une courte période de deux mois.

\section{Conclusion}

Ces limites posées, l'exploration et la mise en perspective des bienfaits potentiels de la pleine conscience et du Tai Chi Chuan sur la santé psychologique d'une population générale d'étudiants apparaissent pour autant heuristiques. Mais la difficulté du recrutement d'étudiants de tous horizons disciplinaires ainsi que la nécessité de leur engagement important dans de tels programmes de huit semaines pèsent sur la faisabilité et la reproductibilité de protocoles semblables. Sur la base de cette étude pilote quasi expérimentale, il ne nous est pas paru possible d'envisager la réalisation d'un protocole expérimental, non seulement contrôlé mais aussi randomisé, qui soit tout autant ajusté au terrain.

À la faveur de ce constat, nous avons essayé de reproduire le même protocole quasi expérimental l'année suivante (2013) pour la même population étudiante, mais sans atteindre un nombre suffisant de participants pour que les résultats puissent être pris en compte. Nous avons pu en revanche engager une recherche similaire, débouchant sur de meilleurs résultats pour la mindfulness, auprès de populations d'enseignants d'écoles primaires ainsi que de collèges et de lycées ; dans le cadre des Plans Académiques de Formation (PAF) du $1^{\text {er }}$ et du

$2^{\text {nd }}$ degré de l'Académie de Limoges. Cette recherche portant sur les enseignants se poursuit. 


\section{Déclaration d'intérêts}

Les auteurs déclarent ne pas avoir de conflits d'intérêts en relation avec cet article.

\section{Références}

[1] Verger P, Guagliardo V, Gilbert F, Rouillon F, Kovess-Masfety V. Psychiatric disorders in students in six French universities: 12-month prevalence, comorbidity, impairment and help-seeking. Soc Psychiatry Psychiatric Epidemiol 2010;45(2):189-99.

[2] $3^{\text {ème }}$ Enquête Nationale sur la Santé des Etudiants. Principaux enseignements. LMDE Santé Des Etudiants 2011, http://www.lmde.com/fileadmin/pdf/securite sociale/ resultats_enquete_ENSE_3.pdf.

[3] Shapiro SL, Shapiro DE, Schwartz GER. Stress Management in Medical Education: A Review of the Literature. Acad Med 2000;75(7):748-59.

[4] Kabat-Zinn J. An outpatient program in behavioral medicine for chronic pain patients based on the practice of mindfulness meditation. Theoretical considerations and preliminary results. Gen Hosp Psychiatry 1982;4:33-47.

[5] Kabat-Zinn J. Mindfulness-based interventions in context: past, present, and future. Clin Psychol: Sci Pract 2003;10(2):144-56.

[6] Kabat-Zinn J. Au cœur de la tourmente, la pleine conscience. MBSR, la réduction du stress basée sur la mindfulness : programme complet en 8 semaines. Bruxelles: De Boeck; 2009.

[7] Brown KW, Ryan RM, Creswell JD. Mindfulness: theoretical foundations and evidence for its salutary effects. Psychol Inquiry 2007;18(4):211-37.

[8] Grossman P, Niemann L, Schimdt S, Walach H. Mindfulness-based stress reduction and health benefits. A meta-analysis. J Psychosom Res 2004;57:35-43.

[9] Hofmann SG, Sawyer AT, Witt AA, Oh D. The effect of mindfulness-based therapy on anxiety and depression: a meta-analytic review. J Consult Clin Psychol 2010;78(2):169_ 83.

[10] Roemer L, Orsillo SM. Expanding our conceptualization of and treatement for generalized anxiety disorder: integrating mindfulness/acceptance-based approaches with existing cognitive-behavioral models. Clin Psychol Sci Pract 2002;9(1):54-68.

[11] Kuyken W, Watkins E, Holden E, White K, Taylor RS, Byford S et al. How does mindfulness-based cognitive therapy work? Behav Res Ther 2010;48(11):1105-12.

[12] van der Velden AM, Kuyken W, Wattar U, Crane C, Pallesen KJ, Dahlgaard J et al. A systematic review of mechanisms of change in mindfulness-based cognitive therapy in the treatment of recurrent major depressive disorder. Clin Psychol Rev 2015;37:26-39. 
[13] Heeren A, Philippot P. Les interventions basées sur la pleine conscience : Une revue conceptuelle et empirique [Mindfulness-based interventions: a conceptual and empirical review]. Rev Que Psychol 2010;31:1-25.

[14] Baer RA. Mindfulness training as a clinical intervention: a conceptual and empirical review. Clin Psychol Sci Pract 2003;10(2):125-43.

[15] Shapiro SL, Carlson LE, Astin JA, Freedman B. Mechanisms of mindfulness. J Clin Psychol 2006;62(3):373-86.

[16] Hölzel BK, Lazar SW, Gard T, Schuman-Olivier Z, Vago DR, Ott U. How does mindfulness meditation work ? Proposing mechanisms of action from a conceptual and neural perspective. Perspect Psychol Sci 2011;6(6):537-59.

[17] Gu J, Strauss C, Bond R, Cavanagh K. How do mindfulness-based cognitive therapy and mindfulness-based stress reduction improve mental health and wellbeing? A systematic review and meta-analysis of mediation studies. Clin Psychol Rev 2015;37:112.

[18] Jain S, Shapiro SL, Swanick S, Roesch SC, Mills PJ, Bell I \& al. A randomized controlled trial of mindfulness meditation versus relaxation training: effects on distress, positive states of mind, rumination, and distraction. Ann Behav Med 2007;33(1):11-21.

[19] Malinowski P. Neural mechanisms of attentional control in mindfulness meditation. Front Neurosci 2013;7:8, http://dx.doi.org/10.3389/fnins.2013.00008.

[20] Chan D, Woollacott M. Effects of level of meditation experience on attentional focus: is the efficiency of executive or orientation networks improved? J Altern Complem Med 2007;13(6):651-57.

[21] Brewer JA, Worhunsky PD, Gray JR, Tang YY, Weber J, Kober H. Meditation experience is associated with differences in default mode network activity and connectivity. Proc Natl Acad Sci USA. 2011;108(50):20254-59.

[22] Fjorback LO, Arendt M, Ornbol E, Fink P, Walach H. Mindfulness-based stress reduction and mindfulness-based cognitive therapy - a systematic review of randomized controlled trials. Acta Psychiatr Scand 2011;124:102-119.

[23] Segal ZV, Williams JMG, Teasdale JD. La thérapie cognitive basée sur la pleine conscience pour la dépression. Une nouvelle approche pour prévenir la rechute. Bruxelles: De Boeck; 2006.

[24] Piet J, Hougaard E. The effect of mindfulness-based cognitive therapy for prevention of relapse in recurrent major depressive disorder: A systematic review and meta-analysis. Clin Psychol Rev 2011;31:1032-40.

[25] Williams JMG, Crane C, Barnhofer T, Brennan K, Duggan DS, Fennell MJV et al. Mindfulness-based cognitive therapy for preventing relapse in recurrent depression: A randomized dismantling trial. J Consult Clin Psychol 2014;82(2):275-86. 
[26] Cottraux J. Thérapie cognitive et émotions. La troisième vague. Issy-les-Moulineaux: Elsevier Masson; 2007.

[27] Trousselard M, Steiler D, Claverie D, Canini F. Pleine conscience, stress et santé [Mindfulness, stress and health]. Rev Que Psychol 2014;35(2):21-45.

[28] Brown KW, Ryan RM. The benefits of being present: mindfulness and its role in psychological well-being. J Pers Soc Psychol 2003;84(4):822-48.

[29] Shapiro SL, Oman D, Thoresen CE, Plante TG, Flinders T. Cultivating mindfulness: effects on well-being. J Clin Psychol 2008;64(7):840-62.

[30] Philippot P, Segal Z. Mindfulness based psychological interventions. Developping emotional awareness for better being. In: Petitmengin $C$, editor. Ten years on viewing from within. The legacy of Francisco Varela. Exeter: Imprint Acad; 2009. p. 285-306.

[31] Berghmans C, Strub L, Tarquinio C. Méditation de pleine conscience et psychothérapie : état des lieux théorique, mesure et pistes de recherche. J Ther Comport Cogn 2008;18(2): 62-71.

[32] Berghmans C, Tarquinio C, Marina K, Strub L. La méditation comme outil psychothérapeutique complémentaire : une revue de question. J Ther Comport Cogn 2009;19(4):120-35.

[33] Gold E, Smith A, Hopper I, Herne D, Tansey G, Hulland C. Mindfulness-Based Stress Reduction (MBSR) for primary school teachers. J Child Family Stud 2010;19(2):18489.

[34] Weare K. Evidence for mindfulness: impacts on the wellbeing and performance of school staff. Mindfulness in schools project, In association with University of Exeter. 2014;10, http://mindfulnessinschools.org/wp-content/uploads/2014/10/Evidence-forMindfulness-Impact-on-school-staff.pdf.

[35] Sanders A. Teachers and mindfulness: a meta-analysis. A thesis presented to the faculty of California State University Stanislaus 2015, https://scholarworks.csustan.edu/ bitstream/handle/011235813/841/SandersA.spring2015.pdf? sequence=1.

[36] Deplus S, Lahaye M, Philippot P. Les interventions psychologiques basées sur la pleine conscience avec l'enfant et l'adolescent : les processus de changement [Mindfulnessbased interventions with children and adolescents: change processes]. Rev Que Psychol 2014;35(2):71-116.

[37] Meiklejohn J, Phillips C, Freedman ML, Griffin ML, Biegel G, Roach A et al. Integrating mindfulness training into K-12 education: fostering the resilience of teachers and students. Mindfulness 2012;3(4):291-307.

[38] Zenner C, Herrnleben-Kurz S, Walach H. Mindfulness-based interventions in schools a systematic review and meta-analysis. Front Psychol 2014;5(603):1-20. 
[39] Shapiro SL, Brown KW, Astin JA. Toward the integration of meditation into higher education: a review of research evidence. Teach Coll Rec 2011;113(3):493-528.

[40] Berghmans C, Tarquinio C, Kretsch M. Impact de l'approche thérapeutique de pleine conscience mindfulness-based stress reduction (MBSR) sur la santé psychique (stress, anxiété, dépression) chez des étudiants : une étude pilote contrôlée et randomisée. J Ther Comport Cogn 2010;20(1):11-15.

[41] Astin JA. Stress reduction through mindfulness meditation. Effects on psychological symptomatology, sense of control, and spiritual experiences. Psychother Psychosom 1997;66:97-106.

[42] Shapiro SL, Schwartz GE, Bonner G. Effects of mindfulness-based stress reduction on medical and premedical students. J Behav Med 1998;21(6):581-99.

[43] Merle Kuhlman SM, Bürger A, Esser G, Hammerle F. A mindfulness-based stress prevention training for medical students (MediMind): study protocol for a randomized controlled trial. Trials 2015;16:40.

[44] Rosenzweig S, Reibel DK, Greeson JM, Brainard GC, Hojat M. Mindfulness-based stress reduction lowers psychological distress in medical students. Teach Learn Med 2003;15(2):88-92.

[45] Ruths FA, de Zoysa N, Frearson SJ, Hutton J, Williams JMG, Walsh J. Mindfulnessbased cognitive therapy for mental health professionals-a pilot study. Mindfulness 2013;4(4):289-95.

[46] Shapiro SL, Brown KW, Biegel GM. Teaching self-care to caregivers: effects of mindfulness-based stress reduction on the mental health of therapists in training. Train Educ Profess Psychol 2007;1(2):105-15.

[47] Crane C, Crane RS, Eames C, Fennell MJV, Silverton S, Williams JMG, Barnhofer T. The effects of amount of home meditation practice in Mindfulness Based Cognitive Therapy on hazard of relapse to depression in the Staying Well after Depression Trial. Behav Res Ther 2014;63:17-24.

[48] Chahine N. Les vrais pouvoirs de la meditation. L'Express 2014;3289:20-36.

[49] Kabat-Zinn J, Santorelli SF, Blacker M, Brantley J, Meleo-Meyer F, Grossman P et al. Training teachers to deliver mindfulness-based stress reduction. Center for Mindfulness. University of Massachusetts Medical School 2015, http://www.umassmed.edu/cfm/training/principles--standards/.

[50] Le Practicum du programme de Réduction du Stress Basée sur la Pleine Conscience (MBSR). Association pour le Développement de la Mindfulness 2015, http://www.association-mindfulness.org/practicum.php.

[51] Booth R. Mindfulness therapy comes at a high price for some, say experts. Guardian 2014; aug(25),http://www.theguardian.com/society/2014/aug/25/mental-healthmeditation. 
[52] Castillo RJ, Depersonalization and meditation. Psychiatry 1990;53(2):158-168.

[53] Teasdale JD, Chaskalson M. How does mindfulness transform suffering? I: The nature and origins of dukkha. Contemp Buddhism 2011;12(1):89-102.

[54] Teasdale JD, Chaskalson M. How does mindfulness transform suffering? II: The transformation of dukkha. Contemp Buddhism 2011;12(1):103-124.

[55] Grabovac AD, Lau MA, Willet BR. Mechanisms of mindfulness: a buddhist psychological model. Mindfulness 2011;2(3):154-166.

[56] Berghmans C, Kretsch M, Branchi S, Strub L, Traquinio C. Effets du taï-chi-chuan sur la santé psychique et physique : une revue systématique de question. J Ther Comport Cogn 2009;19(1):4-29.

[57] Jahnke R, Larkey L, Rogers C, Etnier J, Lin F. A comprehensive review of health benefits of Qigong and Tai Chi. Am J Health Promot 2010;24(6):1-25.

[58] Klein PJ, Adams WD. Comprehensive therapeutic benefits of taiji. A critical review. Am J Phys Med Rehabil 2004;83(9):735-45.

[59] Kuramoto AM. Therapeutic benefits of Tai Chi exercise: research review. Wis Med J 2006;105(7):42-46.

[60] Wang C, Collet JP, Lau J. The effect of Tai Chi on health outcomes in patient with chronic conditions. A systematic review. Arch Int Med 2004;164(5):493-501.

[61] Raman G, Zhang Y, Minichiello VJ, D'Ambrosio C, Wang C. Tai Chi improves sleep quality in healthy adults and patients with chronic conditions: a systematic review and meta-analysis. J Sleep Disord Ther 2013;2(6):141.

[62] Raman G, Mudedla S, Wang C. How effective is Tai Chi mind-body therapy for fibromyalga: a systematic review and meta-analysis. J Altern Complement Med 2014;20(5):A66.

[63] Wang C, Bannuru R, Ramel J, Kupelnick B, Scott T, Schmid CH. Tai Chi on psychological well-being: systematic review and meta-analysis. BMC Complement Altern Med 2010;10:23.

[64] Wei D. Taijiquan exercise and mental health: a meta-analysis. J Fujian Normal Univ 2011;27(5):111-16. (Article written in chinese).

[65] Li G, Yuan H, Zhang W. Effects of Tai Chi on health related quality of life in patients with chronic conditions: A systematic review of randomized controlled trials. Complement Ther Med 2014:22(4):743-55.

[66] Wang WC, Zhang AL, Rasmussen B, Lin LW, Dunning T, Kang SW et al. The effect of Tai Chi on psychosocial well-being: a systematic review of randomized controlled trials. J Acupunct Meridian Stud 2009;2(3):171-81. 
[67] Jimenez PJ, Melendez A, Albers U. Psychological effects of Tai Chi Chuan. Arch Gerontol Geriatr 2012;55(2):460-67.

[68] Yin J, Dishman RK. The effect of Tai Chi and Qigong practice on depression and anxiety symptoms: A systematic review and meta-regression analysis of randomized controlled trials. Ment Health Phys Act 2014;7(3):135-46.

[69] Wang F, Lee EKO, Wu T, Benson H, Fricchione G, Wang W et al. The effects of Tai Chi on depression, anxiety, and psychological well-being: a systematic review and meta-analysis. Int J Behav Med 2014;21:605-17.

[70] Sharma M, Haider T. Tai Chi as an alternative and complimentary therapy for anxiety: a systematic review. J Evid Based Complement Altern Med 2015;20(2):143-53.

[71] Dechamps A, Lafont L, Bourdel-Marchasson I. Effects of Tai Chi exercises on selfefficacy and pyschological health. Eur Rev Aging Phys Act 2007;4:25-32.

[72] Verhagen AP, Immink M, van der Meulen A, Bierma-Zeinstra SMA. The efficacy of Tai Chi Chuan in older adults: a systematic review. Fam Pract 2004;21(1):107-13.

[73] Chi I, Jordan-Marsh M, Guo M, Xie B, Bai Z. Tai Chi and reduction of depressive symptoms for older adults: a meta-analysis of randomized trials. Geriat Gerontol Int 2013;13(1):3-12.

[74] Wayne PM, Walsh JN, Taylor-Piliae RE, Wells RE, Papp KV, Donovan NJ et al. The impact of Tai Chi on cognitive performance in older adults: a systematic review and meta-analysis. J Am Geriatr Soc 2014;62(1):25-39.

[75] Wang YT, Taylor L, Pearl M, Chang LS. Effects of Tai Chi exercise on physical and mental health of college students. Am J Chin Med 2004;32(3):453-59.

[76] Zheng G, Lan X, Li M, Ling K, Lin H, Chen L et al. The effectiveness ot Tai Chi on the physical and psychological well-being of college students: a study protocol for a randomized controlled trial. Trials 2014;15:129.

[77] Dechamps A, Quintard B, Lafont L. Effets d'une intervention courte en Tai-Chi-Chuan sur le sentiment d'efficacité, l'anxiété et les troubles émotionnels chez des étudiants ayant un style de vie sédentaire. Eur Rev Appl Psychol 2008;58(2):125-32.

[78] Yang XQ. Experimental study of the effect of Taijiquan on psychological health of college students. J of TjIPE 2003;18(1):63-66. (Article written in chinese).

[79] Li AT, Sun FC. An experiment research on how Taijiquan improved the undergarduates psychological health. Liaoning Sport Sci Technol 2009;28(5):63-66. (Article written in chinese).

[80] Feng ZQ. Effects of Chen style and Yang style Taijiquan exercise on cardiovascular function of university students. J Zhengzhou Univ 2013;48(2):253-56. (Article written in chinese). 
[81] Zhan JP. Research on the principal influencing factors of Taijiquan teaching in colleges and universities. J Jilin Sport Univ 2013;29(6):81-83. (Article written in chinese).

[82] Sandlund ES, Norlander T. The effects of Tai Chi Chuan relaxation and exercise on stress responses and well-being: an overview of research. Int J Stress Manag 2000;7(2):139-49.

[83] Jin P. Efficacy of Tai Chi, brisk walking, meditation, and reading in reducing mental and emotional stress. J Psychosom Res 1992;36(4):361-70.

[84] Posadzki P, Jacques S. Tai Chi and meditation. A conceptual (re)synthesis? J Holist Nurs 2009;27(2):103-14.

[85] Chung PK, Si GY, Liu JD, Zhang CQ. The reliability and validity of the Chinese Version of Mindful Attention Awareness Scale (MAAS). Chin J Sport Med 2013;32(12):1105-11. (Article written in chinese).

[86] Liu XH, Han KL, Xu W. Effectiveness of mindfulness-based cognitive behavioral therapy on patients with obsessive-compulsive disorder. Chin Ment Health $J$ 2011;25(2):915-920. (Article written in chinese).

[87] Wang SX, Zheng RM, Wu JL, Liu XH. The application of mindfulness-based stress reduction in medicine. Chin J Clin Psychol 2014;22(5)5:947-950,892. (Article written in chinese).

[88] Xu W, Liu X. Mindfulness training enhances individual's well-being: A review. Chin Ment Health J 2013;27(3):197-202. (Article written in chinese).

[89] Yang S, Zhang ZQ. Effect of mindfulness-based cognitive intervention on the stressrelated psychological parameters of elite athletes. Chin J Sport Med 2014;33(3):214223. (Article written in chinese).

[90] Zhu TT. Mindfulness: the core conception of the third wave of behavior therapy. Med Philos. Humanist Soc Med 2010;31(9):32-34. (Article written in chinese).

[91] Callahan S. La recherche clinique en TCC : guide de l'utilisateur. J Ther Comport Cogn 2012;22(4):148-50.

[92] Goldberg DP, Hillier VF. A scaled version of the General Health Questionnaire. Psychol Med 1979;9:139-45.

[93] Pariente PD Challita H, Mesbah M, Guelfi JD. The GHQ-28 questionnaire in French: a validation survey in a panel of 158 general psychiatric patients. Eur Psychiat 1992;7(1): $15-20$.

[94] Cohen S, Kamarck T, Mermelstein R. A global measure of perceived stress. J Health Soc Behav 1983;24:385-96. 
[95] Bruchon-Schweitzer M. Psychologie de la santé. Modèles, concepts et méthodes. Paris: Dunod; 2002.

[96] Koleck M, Quintard B, Tastet S. French validation of the Perceived Stress Scale: the construct validity. In: 16th European Congress of Health Psychology 2002.

[97] Zigmond AS, Snaith RP. The Hospital Anxiety and Depression Scale. Acta Psychiatr Scand 1983;67(6):361-70.

[98] Lepine JP, Godchau M, Brun P, Lempérière T. Evaluation de l'anxiété et de la dépression chez des patients hospitalisés dans un service de médecine interne. Ann Med Psychol 1985;143(2):175-89.

[99] Razavi D, Delvaux N, Farvacques C, Robaye E. Validation de la version française du HADS dans une population de patients cancéreux hospitalisés. Rev Psychol Appl 1989;39(4):295-307.

[100] Schwarzer R, Jerusalem M. Generalized Self-Efficacy scale. In: Weinman J, Wright S, Johnston M. Measures in health psychology: a user's portfolio. Causal and control beliefs. Windsor: Nfer-Nelson; 1995. p. 35-7.

[101]Dumont M, Schwarzer R, Jerusalem M. French adaptation of the General Self-Efficacy Scale. Berlin; 2000, http://userpage.fu-berlin.de/ health/french.htm.

[102] Schwarzer R, Scholz U. Cross-cultural assessment of coping resources: the General Perceived Self-Efficacy Scale. In: Paper presented at the First Asian Congress of Health Psychology 2000.

[103]Blais MR, Vallerand RJ, Pelletier LG, Brière NM. L'échelle de satisfaction de vie : Validation canadienne-française du «Satisfaction With Life Scale». Rev Can Sci Comport 1989;21(2):210-23.

[104]Bradley C. The Weel-Being Questionnaire. In: Bradley C, editor. Handbook of psychology and diabetes: a guide to psychological measurement in diabetes research and practice. Chur, Switzerland: Harwood Academic Publishers; 1994. p. 89-109.

[105] Walach H, Buchheld N, Buttenmüller V, Kleinknecht N, Schmidt S. Measuring mindfulness - the Freiburg Mindfulness Inventory (FMI). Pers Indiv Dif 2006;40:154355.

[106] Trousselard M, Steiler D, Raphel C, Cian C, Duymedjian R, Claverie D et al. Validation of a French version of the Freiburg Mindfulness Inventory - short version: relationships between mindfulness and stress in an adult population. BioPsychoSoc Med 2010;4:8.

[107]Boudoukha AH, Bonnaud-Antignac A, Acier D, Chaillou A. Anxiété et stress : impact de la préparation du concours de pharmacie. J Ther Comport Cogn 2011;21(3):90-96.

[108]Carson JW, Carson KM, Gil KM, Baucom DH. Mindfulness-based relationship enhancement. Behav Ther 2004;35:471-94. 